\title{
The option to wait in collective decisions*
}

\author{
Matthias Messner ${ }^{\dagger}$ \\ Department of Economics \\ Bocconi University
}

\author{
Mattias K. Polborn ${ }^{\ddagger}$ \\ Department of Economics \\ University of Illinois
}

February 13, 2008

\begin{abstract}
We analyze a model in which voters learn over time their preferences regarding an irreversible social decision. Voters can either implement the project in the first period, or they can postpone the decision to the second period. We analyze the effects of different majority rules. We show that individual first period voting behavior may become "less conservative" under supermajority rules, and that it is even possible that a project is implemented in the first period under a supermajority rule that would not be implemented under simple majority rule.

We characterize the optimal majority rule, which is a supermajority rule. We also show that, in contrast to individual investment problems, society may be better off if the option to postpone the decision did not exist.
\end{abstract}

JEL Classification Numbers: D72, D81.

Keywords: Supermajority rules, learning, investment, option value

\footnotetext{
${ }^{*}$ We thank Alessandro Secchi, Guido Tabellini and seminar participants at Bocconi University for helpful comments.

${ }^{\dagger}$ Department of Economics, Bocconi University, Via Salasco 5, I-20136, Milano; email: matthias.messner@uni-bocconi.it

${ }^{\ddagger}$ Department of Economics, University of Illinois, 470D Wohlers Hall, 1206 S. Sixth St., Champaign, IL, 61820; email: polborn@uiuc.edu
} 


\section{Introduction}

In most political economy models, individuals know their preferences over candidates or social actions. In another branch of the literature, individuals know their fundamental preferences, but which action is best suited to implement them depends on an unknown state of the world. The main objective of this type of models is to analyze how individuals can aggregate dispersed information through strategic voting. ${ }^{1}$

In the present paper we focus on a third case that has received little attention so far: Collective decisions under uncertainty when individuals learn about their own preferences over time. In our model, individuals learn over time about their (heterogeneous) preferences regarding an investment project, and have to choose whether to implement it immediately (with just their initial information), or delay the decision. In the latter case, they can either implement it after receiving additional information, or pass on it completely. While investment problems under uncertainty have been analyzed extensively for single decision makers, the new feature of our paper is to investigate the interplay of individual learning and social decisions. Our main focus is twofold: Firstly, we examine the effect of the majority rule on individual voting behavior and social decisions in this framework. From an ex-ante point of view, we show that a supermajority rule dominates simple majority rule with respect to social welfare. Secondly, we analyze the value of the opportunity to learn over time, and show that it can be either positive or negative; this contrasts with unilateral investment projects where the value of waiting is always positive.

More specifically, we consider the following dynamic social investment problem. There are two periods, and society votes in the first period whether to implement or delay the project. If the project is implemented in the first period, all voters have to incur some adjustment cost and then receive individual-specific payoffs from the project. Voters know their own payoffs for the present period, but their payoff next period is a (possibly individual-specific) random variable, learned only at the beginning of the second period. If society chooses to uphold the status quo in the first period, voters decide again next period whether to implement the project then (which, again, gives rise to the respective costs and payoffs). ${ }^{2}$

In this framework, an advantage of delaying investment in the first period is that agents learn more about their payoffs in the next period: There is an "option value of waiting". We analyze how the type of majority rule influences this option value of

\footnotetext{
${ }^{1}$ See, e.g., Austen-Smith and Banks (1996), Feddersen and Pesendorfer (1996), Feddersen and Pesendorfer (1998).

${ }^{2}$ In principle, voters can also, in the second period, decide to get rid of a project that was implemented in the first one. However, since the adjustment cost for such a project was already paid, there are mild conditions under which society will never reverse a previously implemented project.
} 
waiting, and thus, the voting behavior of individuals and the first period implementation decision. Whether a higher majority rule makes implementation in the first period more or less likely depends on two effects that possibly have opposite signs. There is an obvious direct effect of supermajority rules that makes first-period implementation less likely, as more voters have to agree. ${ }^{3}$ The second effect is more subtle: A supermajority rule may decrease or increase the option value of waiting and thus make each individual voter more or less likely to favor a particular project, respectively.

Intuitively, the expected second period payoff for a voter, if the project is delayed in the first period, may go in either direction as the majority rule changes: A higher supermajority rule may increase the risk that a positive average value project is not implemented in the second period, thus diminishing the option value of waiting and making voters keener to implement the project already in the first period. This effect may dominate the direct effect of an increased majority rule (namely, that more votes are required to pass the project in the first period), thus leading to more projects being implemented as the majority threshold increases.

However, the opposite effect is also possible. A higher supermajority rule may decrease the risk that a negative average value project is implemented in the second period, thus increasing the option value of waiting and making voters more reluctant to implement the project already in the first period. This is socially beneficial, as simple majority rule is not sufficiently conservative in this case: Voters with positive first period payoffs, but overall negative expected payoffs, may vote for implementation, out of a (rational) concern that the project would be implemented anyway in the next period. Since the risk that the decision will be adopted against a given voter's will in the future is lower the higher the majority requirement, this phenomenon is less likely to arise under supermajority rules.

We characterize the optimal supermajority rule, if project costs are uniformly distributed ex-ante (i.e., at the constitutional stage). For any number of voters, the optimal supermajority rule in our model is between $7 / 11 \approx 63.6 \%$ and the smallest implementable rule greater or equal to $2 / 3$. (For example, with 5 voters, the smallest rule greater than a $2 / 3$ rule is 4 out of 5 , or $80 \%$. As the number of voters increases, this upper limit of the interval of possibly optimal majority rules converges to $2 / 3$ ). Many supermajority rules observed in reality are indeed in the neighborhood of the interval $[7 / 11,2 / 3]$.

Even for a given project, a transition from simple majority rule to unanimity rule may lead to a strict Pareto improvement. This is the case if, under simple majority rule,

\footnotetext{
${ }^{3}$ By a supermajority rule, we mean a voting rule that specifies that the status quo is only to be changed if a certain proportion of the electorate (greater than the $50 \%$, the "simple majority") votes in favor of change.
} 
there would be a majority of voters who approve immediate implementation; but even those voters would prefer to postpone implementation, if the majority rule is changed to unanimity rule. In contrast, for a given project, a transition from unanimity rule to simple majority rule can never lead to a Pareto improvement.

It is also interesting to analyze the social ex-ante value of the option to wait. In unilateral investment problems, this option value is always nonnegative, and often positive (i.e., a firm's expected discounted profit from an investment opportunity is strictly larger than what it could get if it were forced to either invest immediately, or forgo the investment completely). In contrast, a society might be better off if it is forced to invest either immediately or not at all, rather than having the option of postponing this decision. Indeed, we show that, from an ex-ante point of view (and with uniformly distributed project costs), this is the case even if society chooses the optimal majority rule for the case when waiting is possible.

Our results shed light on an important question in the endogenous determination of institutions: Why do some organizations choose supermajority rules, and which features of decision problems influence this choice? Majority rules within organizations vary considerably, from simple majority rule to unanimity rule. Often, the choice of the majority rule that is to govern future decision making is a contentious issue itself, such as in the recent EU summit, which, in the end, adopted some form of a supermajority rule. Supermajority rules are also used in most countries for a change of the constitution, and, often implicitly, for "normal" legislation. ${ }^{4}$ This paper contributes to the literature on the advantages and disadvantages of different majority rules (discussed in more detail below), by providing a new rationale for supermajority rules. We will discuss the implications of our results in more detail in Section 6 .

Several previous papers have analyzed arguments for supermajority rules from an economic point of view. Buchanan and Tullock (1962) argue for unanimity rule as the suitable rule governing social choices. Under a simple majority rule, a majority of people could be tempted to implement certain projects that are not socially desirable because they can "externalize" part of the cost associated with this project to the losing minority. Under unanimity rule, only Pareto improving projects are implemented. However, Guttman (1998) has argued that the unanimity rule leads to a rejection of many projects that are not Pareto improvements, but nevertheless worthwhile from a reasonable social point of view. Assuming that the social goal is to minimize the sum

\footnotetext{
${ }^{4}$ For example, in parliamentary systems with a strong committee organization, a legislative proposal usually needs the support of both the respective committee and the house. In parliamentary systems with two chambers, certain legislative proposals need the support of both chambers. Tullock (1998), p.216, estimates that legislative rules in the US for changing the status quo are "roughly equivalent to requiring a $60 \%$ majority in a single house elected by proportional representation." See also Diermeier and Myerson (1999).
} 
of both types of mistakes, he shows that, in a symmetric setting, simple majority rule is optimal. Our model is constructed in a way that simple majority rule would also be optimal if voters have to make a once-and-for-all decision about the project in the first period. However, with the option to postpone a decision to the second period, we show that (in the same symmetric setting), a supermajority rule is optimal. ${ }^{5}$

In terms of the dynamic setup, the paper most related to ours is Glazer (1989), in which voters in period 1 choose between i) implementing an irreversible long run project that delivers benefits both in period one and in period two, ii) implementing a short run project, whose costs and benefits accrue in period one and iii) not investing. In cases (ii) or (iii), the electorate has to decide again in period 2 whether to implement a short run project for period two. While each voter's second period benefit is the same as his first period benefit, there is exogenous uncertainty about the outcome of a possible second period election, which is interpreted as generated by turnout uncertainty. Glazer shows that voters exhibit a bias towards implementing the long run project. Two effects drive this result. First, a first period decisive voter with a positive net payoff from the project may prefer to disempower the second period decisive voter (who may have different preferences) by committing to a long-term project that cannot be changed, even if it were cheaper to implement two short term projects. Second, even if the first period decisive voter has a negative net payoff from the long-term project, he may prefer to implement this, if he is sufficiently afraid that a short-term project would be implemented in the second period that would be even worse for him.

Apart from the fact that we have no first period short term project, our analysis differs from Glazer (1989) in two crucial points. First, we explicitly analyze a dynamic voting game in which each voter is uncertain about his second period preferences. This generates endogenous uncertainty about the second period voting outcome, and allows us to analyze how different majority rules affect this uncertainty and hence the first period voting behavior. The focus of our analysis is on the positive and normative implications of different majority rules in dynamic investment problems. Second, while Glazer's second effect is also present in our model for socially undesirable projects, there is not necessarily excessive commitment to early implementation in our model. Indeed, for socially beneficial projects, voters in our model are excessively conservative. Interestingly, in our dynamic model, supermajority rules mitigate both the tendency of voters to excessively commit to socially undesirable projects, and their reluctance to implement socially beneficial projects.

\footnotetext{
${ }^{5}$ Other rationales for supermajority rules are discussed in more detail in Section 5. They include the problem of time inconsistency of optimal policies under simple majority rule (Gradstein (1999), Dal Bo (2006)), the possibility of electoral cycles under simple majority rule (Caplin and Nalebuff (1988)), and a strategic use of supermajority rules in settings with deterministically changing preferences (Messner and Polborn (2004)).
} 
Our model is also related to a small literature in which voters learn about their preferences over time. Strulovici (2007) analyzes a model in which a society has to choose in continuous time between a risky and a secure project. Ex-ante, all individuals are identical; over time, some individuals discover that they are winners and then receive a payoff forever after. The arrival rate is unknown, and voters continuously update their beliefs as long as the risky action is played. In contrast to our model, information arrives only as long as the risky action is played, and the project is reversible. Voters decide under simple majority rule or unanimity rule when (if at all) to stop experimentation with the uncertain action. Strulovici (2007) finds that society always stops experimentation too early compared with a utilitarian optimum, and that unanimity rule may lead to more or less experimentation than simple majority rule.

Fernandez and Rodrik (1991) analyze a model of voting on reform projects that generate winners and losers. They show that a project that ex-post benefits the majority of the population need not be implemented under simple majority rule, if the ex-ante expected benefit is negative for a majority of the population. In a symmetric case (i.e., where a majority of the population has positive ex-ante expected benefits, but ex-post, payoffs are negative for a majority), a reform may be implemented initially, but would be reversed after payoff information becomes known. Thus, there is a bias in favor of the status quo. In contrast to Fernandez and Rodrik (1991), we analyze a setting in which reforms are not reversed, so that there is no "status quo bias" in our setup. Also, our focus is on comparing different majority rules and how they influence voting behavior and implementation decisions, while Fernandez and Rodrik (1991) only consider simple majority rule.

The paper proceeds as follows. In the next section, the model is presented. Our main results are presented in Section 3. In Section 4, we analyze extensions and generalizations of the model. Previous literature is discussed in Section 5, and Section 6 concludes.

\section{The model}

\subsection{Description}

A group of $N$ (odd) risk neutral individuals has to decide whether to undertake an investment project that creates costs and benefits (described in more detail below) for all members of society. The decision about the project has two stages. At the beginning of period 1, the group has to choose between implementing the project right away and postponing the decision to the beginning of period two. In the latter case, the group has to make the final decision on whether or not the project should be implemented at the beginning of period 2 . 
In both periods, the decision is made through a vote governed by a voting rule indexed by $m$, which describes the required majority for implementation. Specifically, the project is implemented if and only if at least $m$ individuals approve. The majority requirement $m$ can take any value in the set $\{(N+1) / 2, \ldots, N\}$, i.e., the voting rule may range from simple majority to unanimity.

If the project is implemented in or before period $t$, then individual $i$ receives a gross payoff of $V_{t}^{i}$ in period $t$. We refer to $V_{t}^{i}$ as $i$ 's type in period $t$. We assume that $V_{t}^{i}$ is either 0 or 1 , each with a probability of $1 / 2$. In addition, the project generates a constant per-period flow of costs, $c \in(0,1)$, for each individual of the society, so that $i$ 's net payoff in period $t$ is $V_{t}^{i}-c .{ }^{6}$ Payoffs are measured relative to non-implementation, that is, in each period that the project is not implemented, each individual receives a net payoff of 0 . For simplicity we assume that individuals do not discount the future, so that they value future and current payoffs equally.

At the moment of the election in period 1, individuals only know their own period1 type, but not their period-2 type. In the period 2 election (if any), individuals know also their period-2 type. In elections, each individual votes for the option that would provide him with the higher expected utility: In the second period, voter $i$ votes for the project if and only if $V_{2}^{i}=1$. In the first period, voter $i$ votes for project implementation if and only if he weakly prefers immediate implementation to the expected payoff from postponing (given that all voters behave in the second period as described in the previous sentence). ${ }^{7}$

Eventually, we will analyze which majority rule is chosen, if the rule $m$ is determined endogenously at an initial stage. Before the type realizations for a project are known, all voters are identical and so all of them agree on the ranking of different majority rules and will unanimously choose the majority rule that maximizes their ex-ante expected payoffs. Note that we can also interpret such a constitution normatively as the one that maximizes ex-ante utilitarian welfare.

In general, ex-ante payoffs and thus the optimal majority rule also depends on the cost parameter $c$. Typically, however, it is not feasible to construct a constitution that specifies a majority rule that depends on the cost parameter of the specific project, since there would be verifiability problems, and such a rule would unavoidably lead

\footnotetext{
${ }^{6}$ Clearly, we could just specify the net payoff of each individual through one variable, but our approach allows us to use $c$ in order to easily distinguish projects with a high expected average payoff (i.e., low $c$ ) from those with a low expected average payoff.

${ }^{7}$ Formally, we use iterated elimination of weakly dominated strategies. This is a standard refinement in voting games. Without it, there are usually multiple equilibria, for example one in which everybody opposes investment, even if we would benefit from implementation. This is an equilibrium because no voter can unilaterally deviate and change the outcome. Elimination of weakly dominated strategies eliminates such strange equilibria.
} 
to conflicts of interpretation. Thus, we focus on the majority rule that is on average optimal, where the expectation is taken both with respect to voter types and cost parameter $c$. We assume that $c$ is drawn from some distribution $f(c)$; throughout most of the paper, we will focus, in particular, on $c$ being drawn from a uniform distribution. ${ }^{8}$

\subsection{Applications}

There are many applications in which societies have to decide on investment projects with uncertain returns. In the following, we present just two examples that illustrate some of the main features and questions of the model. The reader who is more interested to proceed to the theoretical results can, without problems, skip the remainder of this section.

Voting on hiring or promotion. With one small caveat explained below, we can interpret our model as one of hiring by a group that votes on hiring decisions. For example, consider a department that is in the market to hire a junior assistant professors. Suppose that, late in the market, the department can choose to extend an offer to Candidate A, and this candidate would accept an offer, if one is extended. If they choose to do this, the decision is irreversible in the next years (i.e., the line is filled, and the candidate can be fired only after considerable delay at tenure time). Payoffs accrue to department members, both now and in the future, depending on how good a researcher and colleague Professor A turns out to be; from today's perspective, this is a random event, and also a question in which individual tastes of existing department members may differ, so it is plausible that some department members disagree even ex-post whether hiring A was a good decision.

Alternatively, the department can choose to leave the position unfilled and wait till the market of the following year, when there is a new draw of an available candidate, say, B. Again, the department can decide whether to hire B or not. (In principle, the department's hiring problem is an infinite period problem, assuming that their dean would always renew searches for lines that were not filled in the previous year. In the interest of tractability, we simplify the infinite period world to a two-period one in our model.)

There is a small difference between the individuals' payoffs in this application and in our model: In our model, an individual's second period payoff is the same, whether the project was implemented in the first period or only in the second period. In contrast, in this application, an individual voter's realized second period payoff from A (if he

\footnotetext{
${ }^{8}$ Instead of a single project whose parameters are unknown at the constitutional stage, we can also think of many different projects in the future, whose parameters are drawn from the same distribution. The constitution applies to all projects, and is chosen to maximize average payoffs.
} 
was hired in the first period) may very well differ from that individual's payoff from B (in the same period). However, all that matters for individual voters when they decide how to vote in the first period is the expectation of their second period payoff, so the difference between realized payoffs in the model and in the application is immaterial for our main results.

A central result of our model is that supermajority rules outperform simple majority rule with respect to voters' ex-ante expected utility. While we have only anecdotal evidence, supermajority rules appear also prevalent in groups that decide on hiring and/or promotion through voting. ${ }^{9}$ We also show that - in contrast to individual investment problems - the ability to postpone the decision can hurt a society. Thus, groups have an incentive to construct rigid rules in an attempt to commit against reconsideration, if possible. For example, consider tenure decisions. Suppose that a candidate is marginal after six years, say, his publication record is not terrible, but near the borderline, and there is uncertainty about the quality of the candidate's unpublished work. It would appear to be a good idea to postpone the decision on whether to grant tenure for an additional year or two, and only then make the decision. However, university regulations usually preclude such a course of action and force an immediate decision. While such a rigid rule would often lower and never increase the utility of a single decision maker, our model shows that it may be strictly welfare increasing for a group that decides on tenure through voting.

Moving the capital? After German reunification in 1990, the German parliament (Bundestag) had to decide whether or not to transfer the functions of the German capital from Bonn to Berlin. Most votes in the Bundestag split along party lines, but this was not the case for the capital vote. While there were some clearly regional interests (legislators from the Eastern states voting disproportionately for Berlin, and those from the state of Nordrhein-Westfalen voting for Bonn), these regional interest more-or-less neutralized each other, making legislators from the other Western states pivotal. ${ }^{10}$ Many of these legislators had ambivalent feelings about Berlin, based on history (Berlin being the largest German city and historic capital, but also connected to Prussian militarism and the Nazi regime) and relatively weak personal connections to Berlin in the decades of German separation.

Like in our model, the decision was made under uncertainty about several key factors. First, the cost of the move to Berlin was unclear, and there was considerable

\footnotetext{
${ }^{9}$ This is certainly true, as a practical matter, for promotion votes in universities. A candidate who receives a bare majority of favorable votes in his own department usually is in severe problems at the college or university level.

${ }^{10}$ In the current Bundestag, there are 133 deputies from the Eastern part (including Berlin), versus 130 deputies from Nordrhein-Westfalen, and 351 from the other Western states.
} 
uncertainty about the economic development and the general cost of the reconstruction of the formerly communist part of Germany. Conceivably, most legislators would be less enthusiastic about the move to Berlin, if it was clear that the cost of moving were high, or that the general reconstruction costs for the East were high, and vice versa. Second, it was not clear which type of government the new state of Berlin would have in the long-run. The government of the -larger- Western part of the city was led by the conservative CDU before 1990, while the long-run political preferences of many inhabitants of the Eastern part were still unknown in 1991. It appears plausible that a legislator's payoff from moving the capital to Berlin would also be affected by whether or not that city was ruled by a government with which he agreed ideologically.

Since Bonn did not have any constitutional status as German capital in 1991, the decision process in the Bundestag was governed by simple majority rule. Note that, as in our model, a decision to move the capital to Berlin was (practically) irreversible, while the alternative option (i.e., leaving the capital in Bonn) did not impose large up-front costs and could have been reconsidered by the next Bundestag. In the final vote, a majority of 338 to 320 legislators chose to move the capital to Berlin. ${ }^{11}$

Finally, the voting rule had a decisive influence on the outcome, as any supermajority rule above 52 percent would have affirmed the status quo. ${ }^{12}$ It is therefore interesting to analyze the welfare effects of different majority rules, in particular since the majority rule for future changes of this particular issue was later changed to a two-thirds majority. ${ }^{13}$

\section{Results}

\subsection{The benchmark case: No option to wait}

In this section, we analyze the benchmark situation in which a rejection of the project in the first period is final. That is, the electorate has to take the decision about the project once and for all in period one.

Assuming that voters do not play weakly dominated strategies, it follows that each voter approves the project if and only if its net present value is nonnegative. ${ }^{14}$ Voter

\footnotetext{
${ }^{11}$ In a first round, a third proposal of splitting the capital functions between Bonn and Berlin, widely seen as impracticable, failed out.

${ }^{12}$ This argument is a bit loose. As we will see in the following sections, individual voting behavior may depend on the majority rule that governs the decision process. Thus, in principle, the percentage of votes in favor of Berlin might have changed under a different majority rule.

${ }^{13}$ In 1994, the status of Berlin as capital was incorporated into the constitution. Changes of the constitution in Germany require a 2/3 majority in both houses of the German parliament.

${ }^{14} \mathrm{As}$ a tie-breaking assumption, we assume that voters who are indifferent always approve the project. No results of our model qualitatively depend on this assumption.
} 
$i$ 's expected total payoff from immediate implementation, $U_{I}^{i}$, is

$$
U_{I}^{i}\left(V_{1}^{i}, c\right)=V_{1}^{i}+E\left(V_{2}^{i}\right)-2 c=V_{1}^{i}+\frac{1}{2}-2 c .
$$

A voter with first period type $V_{1}^{i}=1$ (a high type) votes in favor of a project with adjustment $\operatorname{cost} c$ if and only if $1+E\left(V_{2}\right)-2 c=3 / 2-2 c \geq 0$, hence if $c \leq 3 / 4$. Similarly, a low type voter $\left(V_{1}^{i}=0\right)$ casts a favorable ballot if and only if $E\left(V_{2}\right)-2 c=1 / 2-2 c \geq 0$, or $c \leq 1 / 4$.

Hence, projects with adjustment cost $c \leq 1 / 4$ are implemented unanimously, and thus it does not matter which majority rule governs the elections. Similarly, projects with adjustment cost $c>3 / 4$ are unanimously rejected. The realization of first period types and the majority rule matter only for $c \in(1 / 4,3 / 4]$, that is, projects with intermediate adjustment costs. For these, the ex-ante probability of implementation (i.e., before nature draws voters' types) decreases in $m$.

As explained in the model section, we assume that the majority rule is chosen to maximize the ex-ante expected payoff of voters. Denote a single voter $i$ 's ex-ante expected payoff under majority rule $m$ when the adjustment cost is $c$ by $\tilde{\pi}_{i}(m, c)$. Given our preceding observations, it follows that if $c \leq 1 / 4$ then $\tilde{\pi}_{i}(m, c)=E\left(V_{1}^{i}\right)+E\left(V_{2}^{i}\right)-$ $2 c=1-2 c$ for all $m$, because the project is unanimously approved, no matter which types are realized. If $c>3 / 4$, then $\tilde{\pi}_{i}(m, c)=0$ for all $m$, because the project is unanimously rejected, no matter which types are realized.

If, instead, $c \in(1 / 4,3 / 4]$ then the realization of types matters. It is useful to define $p(m, N)=2^{-(N-1)}\left(\begin{array}{c}N-1 \\ m-1\end{array}\right)$ as the probability that there are exactly $m-1$ high types among the other $N-1$ voters. We can think of $p(m, N)$ as the probability of voter $i$ being pivotal, if the majority rule is $m$. Also, let $q(m, N)=\sum_{j=m}^{N-1}\left(\begin{array}{c}N-1 \\ j\end{array}\right) 2^{-(N-1)}$ be the probability that there are $m$ or more high types among the other $N-1$ voters. From the point of view of an individual voter $i, q(\cdot)$ is the probability that the project is implemented through the votes of the other voters, independent of what voter $i$ 's preference on the project is.

It is useful to distinguish these two different events (i.e., voter $i$ being pivotal for implementation, and not being pivotal), because they give rise to different conditional expected payoffs from implementation. When there are $m$ or more high types among the other $N-1$ voters, then voter $i$ 's expected payoff is simply the ex-ante expected payoff from implementation, $E\left(V_{1}^{i}\right)+E\left(V_{2}^{i}\right)-2 c$. In contrast, if there are exactly $m-1$ high types among the other $N-1$ voters, then the project is implemented with probability $1 / 2$ (namely, if and only if $i$ 's type is $V_{1}^{i}=1$ ), in which case voter $i$ 's expected implementation payoff over both periods is $1+E\left(V_{2}^{i}\right)-2 c=3 / 2-2 c$. Thus, 
we have

$$
\tilde{\pi}(m, c)= \begin{cases}1-2 c & \text { if } c \leq 1 / 4 \\ q(m, N)(1-2 c)+\frac{p(m, N)}{2}\left(\frac{3}{2}-2 c\right) & \text { if } 1 / 4<c \leq 3 / 4 \\ 0 & \text { if } c>3 / 4\end{cases}
$$

where we have dropped the index $i$, since this payoff is identical for all individuals. We now show that $\tilde{\pi}(m, c)$ is a piecewise linear function of $c$ that jumps downward at $c=1 / 4$ and upward at $c=3 / 4$.

Claim 1. $\tilde{\pi}(m, c)$ is a piecewise linear function of $c$ with $\lim _{c \downarrow 1 / 4} \tilde{\pi}(m, c)<\tilde{\pi}(m, 1 / 4)$ for all $m \leq N$, and $\lim _{c \uparrow 3 / 4} \tilde{\pi}(m, c)<\tilde{\pi}(m, N)$ for all $m<N$.

Proof. Observe that $\lim _{c \downarrow 1 / 4} \tilde{\pi}(m, c)=[q(m, N)+p(m, N)] / 2<\tilde{\pi}(m, 1 / 4)=1 / 2$, and $\lim _{c \uparrow 3 / 4} \tilde{\pi}(m, c)=-q(m, N) / 2$, while $\tilde{\pi}(m, 3 / 4)=0$. Note that, if $m=N$ (i.e., unanimity), then $q(m, N)=0$, so that under unanimity $\tilde{\pi}(m, \cdot)$ exhibits a discontinuity only at $c=1 / 4$ but not at $c=3 / 4$.

Intuitively, at $c=1 / 4$, high types strictly benefit from implementation, while low types are just indifferent. Hence, from an ex-ante perspective, voters strictly benefit if the project is implemented. Implementation always occurs for $c \leq 1 / 4$, while for $c>1 / 4$, implementation depends on the realization of preference types and is thus not guaranteed. For example, under simple majority rule, the probability that a project with any $c \in(1 / 4,3 / 4]$ is implemented is just $1 / 2$. Hence, $\tilde{\pi}(m, c)$ drops at $c=1 / 4$. Similarly, for $c=3 / 4$, high types are just indifferent towards implementation, while low types strictly suffer. Thus, voters suffer from an ex-ante perspective if the project is implemented. Implementation never occurs for $c>3 / 4$ (so that $\tilde{\pi}(m, c)=0$ for all $c>3 / 4)$, while for $c \in(1 / 4,3 / 4]$, implementation depends on the realization of preference types. Figure 1 illustrates the claim and shows the ex-ante payoff for the case $N=7$ and $m=4$ and $m=5$ (the flatter curve between $1 / 4$ and $3 / 4$ ).

Now consider the optimal voting rule for different levels of $c$. First, observe that for $c \leq 1 / 4$ or $c>3 / 4$ the voting outcome is unanimous, and thus payoffs do not depend on the majority rule, so that all majority rules perform equally well.

Second, consider $c \in(1 / 4,1 / 2]$. In this case, both the expected net benefit conditional on being pivotal, $1+E\left(V_{2}\right)-2 c=3 / 2-2 c$ and the unconditional expected net benefit, $E\left(V_{1}\right)+E\left(V_{2}\right)-2 c=1-2 c$, are positive. Since the functions $q(m, N)$ and $p(m, N)$ are both decreasing in $m$, it follows that simple majority is the unique optimal majority rule for all $c \in(1 / 4,1 / 2]$.

Third, consider $c \in(1 / 2,3 / 4]$. In this case, voters can expect to obtain a positive payoff only in case they are pivotal (i.e., $3 / 2-2 c>0>1-2 c$ ). Thus, the optimal 


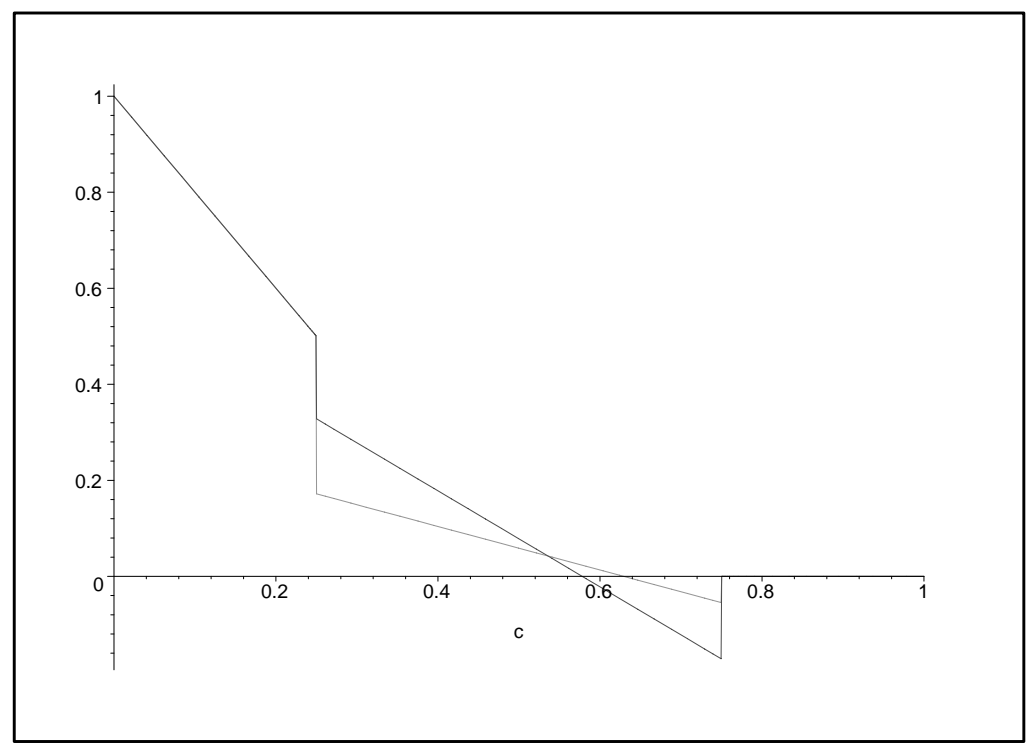

Figure 1: The function $\tilde{\pi}$ for $N=7, m=4$ and $m=5$

majority rule trades off the expected loss if a voter is not pivotal, and the expected gain if he is pivotal. With an increase of $c$, the pivot-benefits decrease while the non-pivot losses increase. To maintain optimality of the majority rule, the relative probability weight on benefits versus losses, $p(m, N) / q(m, N)$, must increase, and this ratio is increasing in $m .{ }^{15}$ Thus, intuitively, the optimal majority rule is increasing in $c$. We now show this formally.

Proposition 1. For $c \in(1 / 2,3 / 4]$, the majority rule that maximizes $\tilde{\pi}(m, c)$ is given by $m^{*}=\left\lceil\left(2 c-\frac{1}{2}\right) N\right\rceil$ (where $\lceil x\rceil$ denotes the smallest integer greater or equal to $x$ ). In particular, for c close to $3 / 4$, the unique optimal majority rule is unanimity rule.

Proof. Since $q(m, N)=q(m+1, N)+p(m+1, N)$, we have that

$$
\begin{aligned}
\tilde{\pi}(m+1, c)-\tilde{\pi}(m, c) & =[q(m+1, N)-q(m, N)](1-2 c)+[p(m+1, N)-p(m, N)]\left(\frac{3}{4}-c\right) \\
& =p(m+1, N)\left(c-\frac{1}{4}\right)-p(m, N)\left(\frac{3}{4}-c\right) .
\end{aligned}
$$

\footnotetext{
${ }^{15}$ We formally show that $p(m, N) / q(m, N)$ is increasing in $m$ in Lemma 2 . The result is particularly intuitive for large $m$ (because $p / q$ goes to infinity for unanimity rule), but is true for every level of $m$ between simple majority and unanimity.
} 
Rearranging, (3) is positive if and only if

$$
c\left[\frac{p(m+1, N)}{p(m, N)}+1\right]>\frac{3}{4}+\frac{p(m+1, N)}{4 p(m, N)} .
$$

Using $p(m+1, N) / p(m, N)=(N-m) / m$, we can conclude that (3) is positive if and only if $m / N<2 c-1 / 2$. Therefore, for $c \in[1 / 2,3 / 4]$, the optimal majority rule is $m^{*}=\left\lceil\left(2 c-\frac{1}{2}\right) N\right\rceil$, as claimed. Substituting shows that unanimity is the unique optimal majority rule for $c \in\left(\frac{3}{4}-\frac{1}{2 N}, \frac{3}{4}\right]$.

The optimal majority rule $m^{*} / N \approx\left(2 c-\frac{1}{2}\right)$ has an intuitive interpretation as the one that maximizes utilitarian welfare: If there are $\hat{M}$ high types in the first period, then the per-capita expected utility from implementation is $\hat{m}-c+\frac{1}{2}-c$ (where $\hat{m}=\hat{M} / N)$. A social planner would like to implement the project if and only if this expected utility is positive, and setting $m^{*}=\left\lceil\left(2 c-\frac{1}{2}\right) N\right\rceil$ guarantees just that.

We now consider the problem of choosing an optimal majority rule when the constitution cannot condition the majority rule for a project on the adjustment cost $c$ of that particular project. We assume that $c$ is distributed symmetrically about $1 / 2$.

Proposition 2. Suppose that society can either implement the investment project in period 1, or not at all. Furthermore, suppose that the constitution cannot condition the majority rule on $c$, and that $c$ is drawn from a distribution $f(\cdot)$ that is symmetric about $1 / 2$. Then simple majority rule maximizes ex-ante expected utility.

Proof. Let $m^{\prime}>\frac{N+1}{2}$ be some supermajority rule, and consider

$$
\begin{aligned}
& \int_{0}^{1}\left[\tilde{\pi}\left(\frac{N+1}{2}, c\right)-\tilde{\pi}\left(m^{\prime}, c\right)\right] f(c) d c= \\
& \int_{1 / 4}^{3 / 4}\left[\left(q(m, N)-q\left(m^{\prime}, N\right)\right)(1-2 c)+\frac{p(m, N)-p\left(m^{\prime}, N\right)}{2}\left(\frac{3}{2}-2 c\right)\right] f(c) d c= \\
& \left(q(m, N)-q\left(m^{\prime}, N\right)\right) \int_{1 / 4}^{3 / 4}(1-2 c) f(c) d c+\frac{p\left(\frac{N+1}{2}, N\right)-p\left(m^{\prime}, N\right)}{2} \int_{1 / 4}^{3 / 4}\left(\frac{3}{2}-2 c\right) f(c) d c>0,
\end{aligned}
$$

because, in the last line, the first integral is equal to zero (due to symmetry), the second is positive (since the integrand is always positive), and $p\left(\frac{N+1}{2}, N\right)>p\left(m^{\prime}, N\right)$ for any $m^{\prime}>(N+1) / 2$. Hence, expected average utility is maximized with a simple majority rule.

The intuition for this result is as follows. In case that a voter is not pivotal for the implementation decision, the expected gains and losses from implementation cancel out for any majority rule, by the symmetry of the distribution of $c$. If the individual is pivotal, then he always receives a positive expected payoff, so the best majority rule from an ex-ante perspective is the one that maximizes the probability of being pivotal, and that rule is simple majority rule. 


\subsection{Individual voting behavior and the option to wait}

We are now ready to analyze the implications of the option to delay the decision on the implementation of the public project. We begin with the straightforward analysis of the voting behavior in second period elections. Player $i$ votes in favor of the project if and only if $V_{2}^{i}=1$, and the project will be implemented if and only if there are at least $m$ players with a high second period type. Let $I_{2}(m)$ denote the event that the project is implemented in the second period, given that a majority of at least $m$ votes is required; and let $P\left(I_{2}(m)\right)$ denote the probability of this event.

Consider now the first period decision. If the project is not implemented in the first period, then player $i$ can expect to obtain the payoff $\left(E\left[V_{2}^{i} \mid I_{2}(m)\right]-c\right) P\left(I_{2}(m)\right)$. It is useful to write this expected continuation utility, the expected value of waiting, as

$$
\begin{array}{r}
U_{W}(c, m)=E\left[\max \left\{V_{2}^{i}-c, 0\right\}\right] p(m, N)+\left(E\left(V_{2}^{i}\right)-c\right) q(m, N)= \\
\frac{1-c}{2} p(m, N)+(1 / 2-c) q(m, N),
\end{array}
$$

using the definitions of $p(\cdot)$ and $q(\cdot)$ from the last section. Since voter $i$ 's payoff from implementing the project immediately is $U_{I}^{i}\left(V_{1}^{i}, c\right)=V_{1}^{i}+1 / 2-2 c$, he will approve immediate implementation in period 1 if and only if

$$
V_{1}^{i}+1 / 2-2 c \geq \frac{1-c}{2} p(m, N)+(1 / 2-c) q(m, N) .
$$

Note an important difference to the benchmark case without the option to wait: An individual voter's first period behavior as characterized by (5) depends on the majority rule $m$, because that rule determines the expected value of waiting.

If $c \leq 1 / 2$, then both terms on the right-hand side of (5) are positive. Thus, in this case, the option to wait induces voters to behave more conservatively than in situations where the decision may not be delayed. Moreover, since both $p(\cdot)$ and $q(\cdot)$ are decreasing functions of $m$, this tendency to behave more conservatively, is the stronger the lower the majority rule $m$. Thus, the cost threshold below which a low voter type is willing to approve a project shifts to the left as the option to delay the decision is introduced, and this shift is the stronger, the lower the majority rule. ${ }^{16}$

If $c>1 / 2$, that is, the project is characterized by a low expected net benefit, then the value of waiting is neither necessarily positive, nor is it necessarily decreasing in $m$. The reason for why the value of waiting can be negative in social decisions - in contrast to private decisions, where the value of waiting is always positive - is that society sometimes implements projects that, from a social ex-ante point of view, are not beneficial. If the right-hand side of (5) is negative, then it is possible that a high

\footnotetext{
${ }^{16}$ For $c \leq 1 / 2,(5)$ implies that high types always favor implementation, so that their behavior does not change relative to the case that waiting is not possible.
} 
type voter votes for immediate implementation of an investment project even though his expected overall payoff from this project is negative. The reason for this (seemingly strange) behavior is that the voter's payoff from immediate implementation is at least better than the expected payoff he would get if he forgoes immediate implementation and is then (perhaps) hit by implementation in the second period, when his type may be low. In this case, a higher supermajority rule may increase the value of waiting, as it increases the voters' protection in the next period against the implementation of a project that they oppose.

We now proceed to a more formal analysis of the value of waiting and its implications for individual voting behavior. Lemmas 1-3 are used repeatedly in the proofs of the following propositions, but are presented here in the text, because they are of independent interest and provide an intuition for the economic effects in our model.

Lemma 1. The value of waiting $U_{W}(c, m)$ defined in equation (4) is decreasing in $m$ if $c<m / N$, and increasing in $m$ if $c>m / N$.

Proof. See Appendix.

Intuitively, for relatively low values of $c$, implementation of the project is socially valuable (taking expectation over type realizations). An increase in $m$ makes second period implementation less likely, thereby decreasing the value of waiting. In contrast, for high values of $c$, an increase in $m$ prevents implementation in some cases where it is socially undesirable (from an ex-ante perspective), and therefore increases the value of waiting. Lemma 1 shows, in particular, that, for any project with $c \geq \frac{N+1}{2}$, the value of waiting is higher for some supermajority rule than for simple majority rule.

Lemma 2 shows that, when we increase the majority rule $m$, then the probability of voter $i$ being pivotal, relative to the probability that the project is implemented independent of voter $i$ 's preferences, increases.

Lemma 2. The ratio $\frac{p(m, N)}{q(m, N)}$ is increasing in $m$.

Proof. See Appendix.

The next lemma shows that, if the value of waiting is negative for some majority rule $m$, then it is also negative for all lower majority rules. Thus, the value of waiting changes its sign at most once.

Lemma 3. If $U_{W}(c, m)<0$, then $U_{W}\left(c, m^{\prime}\right)<0$ for all $m^{\prime}<m$.

Proof. See Appendix. 
Clearly, under unanimity rule, the value of waiting is positive for any $c$ (as $q(N, N)=$ $0)$. Thus, Lemma 3 implies that, for any project with $c>1 / 2$, there exists a critical majority rule threshold such that the value of waiting is positive if and only if the majority rule is above the critical majority rule threshold.

We now characterize individual voting behavior in a situation where the decision on the project may be postponed.

Proposition 3. 1. For any majority rule $m$ there exist threshold values $1 / 12 \leq$ $\underline{c}(m)<1 / 4$ and $2 / 3<\bar{c}(m)<5 / 6$ such that in period one the project is approved (rejected) by all voters (i.e. independently of their payoff type) iff $c \leq \underline{c}(m)$ $(c>\bar{c}(m))$. If $\underline{c}(m)<c \leq \bar{c}(m)$ then the project is approved only by voters with a high first period payoff type.

2. The function $\underline{c}(\cdot)$ is strictly increasing in $m$. In contrast, $\bar{c}(\cdot)$ is $U$-shaped and assumes its minimum at $m=\lceil 3 N / 4\rceil$. Moreover, $\bar{c}((N+1) / 2)>\bar{c}(N)$.

Proof. We start by showing that the two thresholds lie within the claimed bounds. Consider first the threshold value for low payoff types, $\underline{c}(m)$. If $c=1 / 4$, then the payoff of an immediate implementation of the project for low type voters is given by substituting in (1), $U_{I}(0,1 / 4)=E\left[V_{2}\right]-2 \cdot(1 / 4)=1 / 2-1 / 2=0$. The payoff from waiting is $U_{W}(1 / 4, m)=(3 / 8) p(m, N)+(1 / 4) q(m, N)$, which is positive for all $m$. Therefore, low type voters always reject the project in period one if $c \geq 1 / 4$, i.e. $\underline{c}(m)<1 / 4$.

At $c=1 / 12$, we have $U_{I}(0,1 / 12)=E\left[V_{2}\right]-2 \cdot(1 / 12)=1 / 3$, and $U_{W}(1 / 12, m)=$ $(11 / 24) p(m, N)+(5 / 12) q(m, N)=(p(m, N) / 2+q(m, N))(5 / 12)+p(m, N) / 4$. Given that both $p(m, N) / 2+q(m, N)$ and $p(m, N)$ each cannot be larger than $1 / 2$, it follows that $U_{W}(1 / 12, m)<5 / 24+1 / 8=1 / 3$. Hence, a low type voter strictly favors immediate implementation of a project with $c=1 / 12$. Thus, $1 / 12 \leq \underline{c}(m)<1 / 4$

As for the threshold $\bar{c}(m)$, notice that implementing the project right away for $c=2 / 3$ and $c=5 / 6$ yields to high type voters a payoff of $U_{I}(1,2 / 3)=3 / 2-$ $2(2 / 3)=1 / 6$, and $U_{I}(1,5 / 6)=3 / 2-2(5 / 6)=-1 / 6$, respectively. The corresponding expected payoffs from waiting are $U_{W}(2 / 3, m)=(1-2 / 3) p(m, N) / 2+(1 / 2-$ $2 / 3) q(m, N)=(p(m, N)+q(m, N)) / 6<1 / 6$ and $U_{W}(5 / 6, m)=(1-5 / 6) p(m, N) / 2+$ $(1 / 2-5 / 6) q(m, N)=p(m, N) / 12-q(m, N) / 3>-q(m, N) / 3>-1 / 6$. Thus, if $c \leq 2 / 3$, high types always favor immediate implementation, and if $c \geq 5 / 6$, they always reject immediate implementation. Thus, $\bar{c}(m) \in(2 / 3,5 / 6)$.

The proof of the second part is in the appendix. 


\subsection{Ex ante payoffs under different majority rules}

The key result of Proposition 3 is how the majority rule influences individual voting behavior. For low cost projects, individual voters behave more conservatively under lower majority rules. In contrast, for high cost projects, individual voters behave more conservatively under higher majority rules. We will now analyze the implications for voters' ex-ante payoffs.

We denote a player's ex-ante payoff, that is, his expected payoff given majority rule $m$ and implementation cost $c$, but before the player's type is known, by $\pi(m, c)$. For $c \leq \underline{c}(m)$, all voters always approve the first-period implementation of the project, so that we have $\pi(m, c)=\tilde{\pi}(m, c)=1-2 c$. If $c>\bar{c}(m)$, then all voters reject the project in the first period and so $\pi(m, c)$ in this case simply coincides with the value of waiting, $U_{W}(c, m)=q(m, N)(1 / 2-c)+p(m, N)(1-c) / 2$. Finally, if $c \in(\underline{c}(m), \bar{c}(m)]$, then the project is approved in period 1 if and only if there are sufficiently many high type voters in period 1. In contrast to Section 3.1, after a first period rejection (which occurs with probability $[1-q(m, N)-p(m, N) / 2])$, the project may still be implemented in period 2, so that $\pi(m, c)=\tilde{\pi}(m, c)+[1-q(m, N)-p(m, N) / 2] U_{W}(c, m)$. Rearranging terms and dropping the arguments from the functions $q$ and $p$, we thus have

$\pi(m, c)= \begin{cases}1-2 c & \text { if } c \leq \underline{c}(m) \\ q(1-2 c)+\frac{p}{2}\left(\frac{3}{2}-2 c\right)+\left(1-q-\frac{p}{2}\right)\left[q\left(\frac{1}{2}-c\right)+\frac{p}{2}(1-c)\right] & \text { if } c \in(\underline{c}(m), \bar{c}(m)] \\ q\left(\frac{1}{2}-c\right)+\frac{p}{2}(1-c) & \text { if } c>\bar{c}(m) .\end{cases}$

It is easy to see that a result parallel to Claim 1 obtains: For any $m$, the ex-ante payoff $\pi(m, \cdot)$ is a piecewise linear function of $c$ that exhibits a downward jump at $\underline{c}(m)$, and, unless $m=N$, an upward jump at $\bar{c}(m)$. Figure 2 depicts the ex ante payoff for the cases $m=8$ (black curve) and $m=9$ (blue curve).

We now turn to an analysis of the optimal majority rule for a given level of $c$. The ranking of majority rules in the case that society can choose to delay implementation may be markedly different from the ranking in the benchmark case of Section 3.1.

There are several different effects. First, consider projects that are rejected by all voters in the first round (i.e., with $c>\bar{c}((N+1) / 2))$. In contrast to Section 3.1, these projects may now still be approved in the second period. Since those projects are projects with a very low expected net social benefit, it follows that higher majority rules outperform lower majority rules, since they decrease the probability that such projects will pass.

Second, the cost threshold below which high type voters are willing to implement projects in period 1 now depends on the majority rule. In a sense, under low majority rules, introducing the option to wait reinforces the electorate's tendency to behave too 


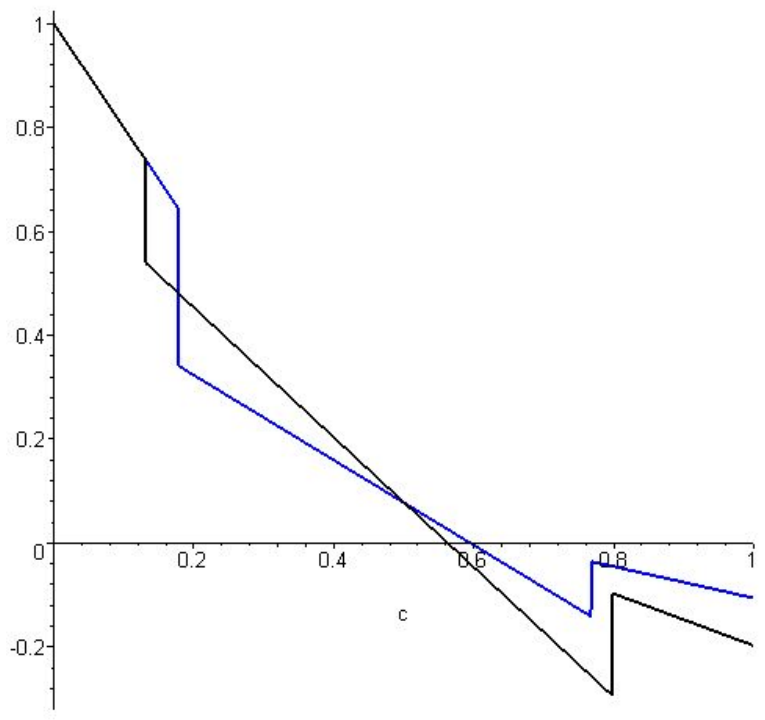

Figure 2: The function $\pi$ for $N=15, m=8$ (black) and $m=9$ (blue)

'aggressively' in implementing projects with low expected net benefits. Specifically, take a setting where $\bar{c}(m+1)<\bar{c}(m)$, and consider a project with a value of $c$ that lies in between these two thresholds. Majority rule $m+1$ guarantees that any project with a cost parameter in $(\bar{c}(m+1), \bar{c}(m)]$ is at least not implemented in period 1 . This is clearly beneficial if the project's expected net benefit is negative for both groups, i.e. if $3 / 4<\bar{c}(m+1)$. As shown in the proof of Proposition 3, $\bar{c}(m+1)=(3-q(m+1, N)+$ $p(m+1, N)) /(4-2 q(m+1, N)-p(m+1, N))$ and thus $\bar{c}(m+1)>3 / 4$ whenever $2 q(m+1, N) \leq p(m+1, N)$. Since $q(m+1, N)>p(m+2, N)$ and $p(m+2, N)=$ $(N-m-1) p(m+1, N) /(m+1)$ it therefore follows that $\bar{c}(m+1)>3 / 4$ whenever $(m+1) / N \leq 2 N / 3{ }^{17}$ In this case, the higher majority rule $m+1$ leads to a higher ex-ante payoff than the majority rule $m$.

It might even be true that, after the first period types are realized, voters would unanimously agree to change the majority rule. Consider, for example, a society with simple majority rule, a project with $c$ between $3 / 4$ and $\bar{c}((N+1) / 2)$, and a majority of high types. Without a change in the majority rule, the project is implemented by the

\footnotetext{
${ }^{17}$ The condition $m / N \leq 2 / 3$ is a rather 'loose' sufficient condition for $\bar{c}(m) \geq 3 / 4$, since it only uses $q(m, N)>p(m+1, N)$. Of course, $q(m, N)=\sum_{l=m+1}^{N-1} p(l, N)$. Notice that if $N$ is sufficiently large this sum has more than one component if $m / N \leq 3 / 4$ and the more of them we take into account in our argument the farther to the right we can shift the lower bound on $m / N$. In particular, using $q(m, N)>p(m+1, N)+p(m+2, N)$ it can be shown that $\bar{c}(m) \geq 3 / 4$ whenever $(1-s) / s \geq(s N+1) / 2 N$, where $s=m / N$. The rhs of this expression converges to $s / 2$ as $N$ grows. Thus, for large $N$ this inequality is essentially equivalent to $2(1-s) \geq s^{2}$ which is satisfied for all $s \leq \sqrt{3}-1 \approx 0.732$. This suggests that $\bar{c}(\cdot)$ crosses $3 / 4$ at a majority thresholds just below $3 N / 4$.
} 
support of all high types in the first period. However, all voters (including first period high types) would be better off if society switched to unanimity rule, thereby killing the project in the first period. Thus, a change from simple majority rule to unanimity rule leads to an ex-post Pareto improvement in this example.

Another interesting effect arises in the case of projects with high expected net benefits. Since $\underline{c}(m)<\underline{c}(m+1)$, projects in the interval $(\underline{c}(m), \underline{c}(m+1))$ are approved in the first period for sure under majority rule $m+1$, while they may be rejected under majority rule $m$. As $\underline{c}(m+1)<1 / 4$, these projects are characterized by high expected social benefits. Thus, from an ex-ante perspective, voters are better off if such projects are more likely to pass. Again, this effect favors higher majority rules.

We summarize these observations in the following proposition.

Proposition 4. 1. For $c \leq \underline{c}((N+1) / 2)$, all majority rules always lead to first period implementation, and thus, the expected ex-ante payoff is the same under any majority rule.

2. For $c \in(\underline{c}((N+1) / 2), \underline{c}(N))$, unanimity is an optimal majority rule. In particular, it strictly dominates simple majority rule.

3. Unanimity rule also dominates simple majority for $c>3 / 4$.

We now turn to a different set of effects that increase the benefit of low majority rules. Because all thresholds $\underline{c}$ decrease below $c=1 / 4$, the behavior of the electorate becomes more conservative under any majority rule. Of course, for $c \in(\underline{c}(N), 1 / 4)$ the negative welfare consequences of this effect are less severe with a lower majority rule. Moreover, for projects with $c \in(1 / 4,1 / 2)$ the fact that the project may still be implemented in period two, even if it is rejected in period one, reinforces the relative advantage of low majority rules. Clearly, to this stronger relative advantage corresponds a larger comparative disadvantage for projects with negative expected net benefits.

\subsection{Average ex-ante payoffs}

The option to wait produces effects both in favor and against low majority rules, so it is natural to ask which of these effects dominate and whether, as in Proposition 2, simple majority remains the optimal majority rule in terms of average ex-ante payoffs. It turns out that this is not the case, if we assume that the adjustment costs of projects are uniformly distributed over the unit interval.

Proposition 5. Let $\Pi(m)=\int_{0}^{1} \pi(m, c) d c$.
i) $\Pi(m+1)-\Pi(m)<0$ for all $m \geq 2 N / 3$ and
ii) $\Pi(m+1)-\Pi(m)>0$ for all $(N+1) / 2 \leq m<7 N / 11$. 
Moreover, $\Pi((N+1) / 2)<\Pi(N)$.

Proof. See Appendix.

While Proposition 5 does not determine the optimal majority rule exactly, it is clear from (i) that the optimal majority rule is a supermajority rule with $m / N \geq 7 / 11 \approx$ 0.636. From (ii), it follows that the optimal majority rule is at most $\lceil 2 N / 3\rceil$, i.e., the lowest majority rule that is higher than a two-thirds majority. In particular, if the number of voters $N$ is large, then the optimal majority rule as a percentage of the electorate lies either within or arbitrarily close to the interval $[7 / 11,2 / 3]$.

Quite interestingly, when the option to wait is introduced, simple majority not only loses its status as the optimal majority rule, but it actually becomes the worst majority rule. It is dominated even by unanimity (which is the worst of all supermajority rules that have $m \geq\lceil 2 N / 3\rceil$. Thus, loosely speaking, erring on the side of choosing a "too high" supermajority rule has a lower welfare cost than choosing a majority rule that is "too low".

Some intuition for why supermajority rules dominate simple majority rule can be gained by considering the case of many voters $(N \rightarrow \infty)$. Since the proportion of high types among all voters is almost certainly close to $1 / 2$, the project will not be implemented in the second period under any supermajority rule. Thus, the value of waiting is zero. Furthermore, a project is implemented in the first period if and only if low types agree, that is, if $\frac{1}{2}-2 c \geq 0$. Hence, all projects with $c \leq 1 / 4$ are implemented in the first period, and no projects with $c>1 / 4$ are ever implemented.

Now consider simple majority rule. In the second period, all projects are implemented with probability $1 / 2$, and so, for given $c$, the value of waiting is $U_{W}=\frac{1}{2}\left(\frac{1}{2}-c\right)$. Thus, in the first period, low types agree if and only if $\frac{1}{2}-2 c \geq \frac{1}{2}\left(\frac{1}{2}-c\right)$, hence whenever $c \leq 1 / 6$; similarly, first period high types vote for immediate implementation whenever $c \leq 5 / 6$. Thus, if $c \leq 1 / 6$, the project is implemented in the first period. Projects with $c \in(1 / 6,5 / 6]$ are implemented with probability $1 / 2$ in the first period, and projects with $c>5 / 6$ are not implemented in the first period.

Because project costs are drawn from a uniform distribution, the average net value of projects from the middle interval, $c \in(1 / 6,5 / 6]$, is zero. Moreover, given that all projects with $c \leq 1 / 6$ are already implemented in the first period, the average cost, conditional on the event that a project was not implemented in the first period, is larger than $1 / 2$, and thus the expected average payoff from a project that is implemented under simple majority rule in the second period is negative.

In summary, there are two reasons for why simple majority rule is dominated by (any) supermajority rule in this setting. First, fewer good projects are implemented with certainty in the first period under simple majority rule than under any super- 
majority rule (only those with $c \leq 1 / 6$, rather than those with $c \leq 1 / 4$ ). Second, those projects that are implemented under simple majority rule only in the second period have, on average, a negative social value, while, under any supermajority rule, no projects are ever implemented only in the second period.

Nothing in this intuitive argument (for the case that $N$ is large) relies on the cost distribution being specifically uniform. The argument would still hold if the cost distribution were symmetric around $1 / 2$, and realizations between 0 and $1 / 4$ had a positive probability. However, for finite $N$, and cost distributions that have a very high probability mass clustered in a neighborhood of $1 / 2$, it is possible that simple majority rule is optimal. This is why in Proposition 5 we have to make a stronger assumption on the distribution of $c$ than in Proposition 2

Note that all supermajority rules yield approximately the same payoff for the case that the number of voters $N$ is large. Thus, this case cannot be used to gain intuition for why the optimal supermajority rule is greater than $\frac{7}{11} N$, and the reader is referred to the appendix for a formal proof of that result.

\subsection{Does the option to wait increase the welfare of voters?}

In settings with a single decision maker, the option to delay the investment decision always weakly increases the decision maker's expected profit. This is obvious, as the decision maker can still go ahead and invest immediately, if he chooses to do so, but sometimes the option to wait is valuable for the decision maker. While the setup of our social decision problem is similar, the answer to the title question is not obvious, as the option to wait influences a game between different voters (rather than the decision problem of a single decision maker).

Indeed, for some values of $c$, the option to wait hurts all citizens from an exante perspective. For example, projects with $c>3 / 4$ are never implemented in a setting without the option to wait, as even the first period high types have a negative expected profit from their implementation. In contrast, with the option to wait and simple majority rule, each project that was rejected in the first period has a 50 percent chance of being accepted in the second round; in most of the cases when the project is implemented in the second period, the percentage of winners is smaller than $c$, and so the project is not desirable from an ex-ante point of view. Moreover, since $\bar{c}(m)>3 / 4$ for many majority rules, it is also the case that some projects that would definitely be rejected without the option to wait are actually implemented in the first period with positive probability, if the option of waiting exists.

On the other hand, there are also project types for which the option of waiting is beneficial for expected social welfare. For instance, if $c \in(\underline{c}(m), 1 / 2)$, then a project may be rejected in the first period. Without the option to wait, such a rejection is 
final, while there is a second period chance for (on average beneficial) implementation with the option to wait.

Thus, there exist some cost levels for which ex-ante welfare increases with the option to wait, and others where that welfare decreases with the option to wait. Again, it is interesting to see which effect dominates when project costs are drawn from a uniform distribution.

Proposition 6. If $N>3$ and $m \leq\lfloor 3 N / 4\rfloor$ then $\tilde{\Pi}(m, N)>\Pi(m, N)$. If, instead, $m \geq 13 N / 16$ then $\tilde{\Pi}(m, N)<\Pi(m, N)$.

Proof. See Appendix.

Proposition 6 shows that, for a large range of low supermajority rules, the option to wait is, in expectation, harmful for voters. Only under very high majority rules, the option to wait is guaranteed to have a positive social value in terms of average ex-ante payoffs.

Combining this result with Proposition 5 allows us to draw an even stronger conclusion: The expected ex-ante payoff with the optimal majority rule under the option of waiting is lower than the expected ex-ante payoff with the optimal majority rule without the option of waiting, if $N>5$. This follows, because, by Proposition 5 , the optimal majority rule with the option of waiting is lower or equal to $\lceil 2 N / 3\rceil$, which is lower or equal to $\lfloor 3 N / 4\rfloor$ for all $N>5$. By Proposition 6 , for all such rules, the expected ex-ante payoff is higher without the option to wait.

Corollary 1. For $N>5$, the maximal average ex-ante payoff strictly decreases due to the introduction of the option to wait. That is, $\max _{m} \tilde{\Pi}(m)>\max _{m} \Pi(m)$.

In a strong sense, having the option of waiting makes society on average worse off, whereas an individual decision maker would be strictly better off.

Again, it is instructive to think about the intuition for this result in a setting where $N$ is large. Under simple majority rule without the option to wait, all projects with $c \leq 1 / 4$ are unanimously implemented, just like under the optimal supermajority rule. In addition, however, projects with $c \in(1 / 4,3 / 4]$ are implemented under simple majority rule if and only if a majority of voters has a high type, and this is, on average, better (from an ex-ante perspective) than not implementing any of these projects. ${ }^{18}$

\section{Extensions}

Like most political economy models, our model imposes some strong structural assumptions. In particular, we assume that there are only two different payoff types in each

\footnotetext{
${ }^{18}$ Clearly, this argument requires that $N$ is large, but finite, because when we take $N$ to infinity, then $\lim \Pi(m)=\lim \tilde{\Pi}(m)$.
} 
period, types are equally likely, and the second period type of a voter is independent of his first period type. We do this in order to generate tractability and comparability to the case without the option to wait. For example, there is no particular reason to assume that $c$ is drawn from a uniform distribution (rather than some other distribution on $[0,1])$. However, this structure is tractable and guarantees sufficient symmetry to ensure that, without the option to wait, simple majority rule is optimal. We can therefore be sure that a result like the optimality of a supermajority rule in Proposition 5 is driven by the option value of waiting.

In this section, we want to explore the robustness of the model when we loosen some of our assumptions. In Section 4.1, we analyze a setting where second period valuations of individuals are correlated with each other, so that voters are more likely to agree with the majority of other voters ex-post. In Section 4.2, the first- and second-period valuations of each individual are correlated, that is, first period high types are more likely to be second period high types than first period low types.

\subsection{Systematic second-period risk}

In our basic model, the probability of being a high type in the second period is $1 / 2$ for every voter. Here, we assume instead that, at the beginning of the second period, nature draws a parameter $\mu$ from a uniform distribution on $[0,1]$; then, each voter is assigned a high type with probability $\mu$ (and, correspondingly, a low type with probability $1-\mu$ ).

Note that this variation of the model would not at all affect the expected utility or first period actions of a single decision maker, as, from the perspective of the first period, the expected probability of being a high type in period 2 remains at $1 / 2$ in this scenario. Consequently, both the implementation payoff and the value of waiting remain unchanged.

However, the model variation introduces correlation between the types of different voters in the second period: The probability of voter $i$ being a high type in the second period, conditional on voter $j \neq i$ being a high type in that period, is $\operatorname{Prob}\left(V_{2}^{i}=\right.$ $\left.1 \mid V_{2}^{j}=1\right)=\frac{\operatorname{Prob}\left(V_{2}^{i}=1 \cap V_{2}^{j}=1\right)}{\operatorname{Prob}\left(V_{2}^{j}=1\right)}=\frac{\int_{0}^{1} \mu^{2} d \mu}{\int_{0}^{1} \mu d \mu}=2 / 3$, while this probability is equal to $1 / 2$ in the basic model. Effectively, while voters still do not know in the first period whether they will like the project in the second period, they are now more likely than in the basic model to agree with the majority of the other voters about the desirability of the project ex-post: Say, if $\mu$ turns out to be high, then it is likely that a particular voter $i$ is a high type, and also likely that the majority of other voters agrees.

For example, consider the job market example from Section 2.2. Suppose that, whether a particular faculty member "likes" next year's candidate (i.e., receives a positive net payoff from the candidate being hired) depends stochastically on that candidate's "quality" (i.e., $\mu$ ). A high quality candidate is more likely to be liked by each 
existing faculty member than a low quality candidate, so there is correlation between the opinions of different voters. However, if the department rejects this year's candidate, its voters do not know the quality of the (feasible) candidates next year, so next year's $\mu$ is a random variable from today's perspective.

What is the value of waiting in this setup, for a project with a given value of $c$ ? Just as in the basic model, we can condition on whether voter $i$ is or is not pivotal for second period implementation. Writing $p_{2}(m, N, \mu)$ and $q_{2}(m, N, \mu)$ for the obvious generalizations of the functions $p(m, N)$ and $q(m, N)$ from the basic model, we have

$$
\begin{aligned}
& U_{W}(c, m, N)=E\left[\max \left\{V_{2}^{i}-c, 0\right\}\right] p(m, N, \mu)+\left(E\left(V_{2}^{i}\right)-c\right) q(m, N, \mu)= \\
& \int_{0}^{1}\left[\left(\begin{array}{l}
N-1 \\
m-1
\end{array}\right) \mu^{m-1}(1-\mu)^{N-m} \cdot \mu(1-c)+\sum_{k=m}^{N-1}\left(\begin{array}{c}
N-1 \\
k
\end{array}\right) \mu^{k}(1-\mu)^{N-1-k} \cdot(\mu-c)\right] d \mu .
\end{aligned}
$$

The first term in (7) refers to the case that individual $i$ is pivotal, which happens with probability $\left(\begin{array}{l}N-1 \\ m-1\end{array}\right) \mu^{m-1}(1-\mu)^{N-m}$; then, with probability $\mu$, individual $i$ is a high type and votes for implementation, in which case his payoff is $1-c$. The second term refers to the case that there are at least $m$ high types among the other voters; in this case, voter $i$ 's expected type is $\mu$, so that his expected payoff from implementation is $(\mu-c)$.

Just like in the basic model, intersecting $U_{W}(c, m, N)$ with $U_{I}(0, c)$ and with $U_{I}(1, c)$ yields the values for $\underline{c}(m, N)$ and $\bar{c}(m, N)$, respectively. All projects with $c \leq \underline{c}(m, N)$ are unanimously approved in the first period; all projects with $c>\bar{c}(m, N)$ are unanimously rejected in the first period; and for projects with $c \in(\underline{c}(m, N), \bar{c}(m, N)]$, voting is type dependent and thus, whether the project is implemented in the first period depends on the realization of first period types.

Observe that the function $U_{I}\left(V_{1}, c\right)$ is linear in $V_{1}$, so $E_{V_{1}} U_{I}\left(V_{1}, c\right)=U_{I}\left(E\left(V_{1}\right), c\right)$. Given this, we can write the ex-ante expected utility, given majority rule $m$, as

$$
\begin{aligned}
& \Pi(m, N)=\int_{0}^{\underline{c}(m, N)} U_{I}(0.5, c) d c+\int_{\bar{c}(m, N)}^{1} U_{W}(c, m, N) d c+ \\
& \int_{\underline{c}(m, N)}^{\bar{c}(m, N)}\left[\sum_{k=m}^{N}\left(\begin{array}{l}
N \\
k
\end{array}\right)\left(\frac{1}{2}\right)^{N} U_{I}\left(\frac{k}{N}, c\right)+\left(1-\sum_{k=m}^{N}\left(\begin{array}{c}
N \\
k
\end{array}\right)\left(\frac{1}{2}\right)^{N}\right) U_{W}(c, m, N)\right] d c
\end{aligned}
$$

The first of these terms corresponds to those projects that have $c \leq \underline{c}(m, N)$, which are all implemented in the first period. All projects with $c>\bar{c}(m, N)$ are delayed in the first period with probability 1 , and each voter obtains the value of waiting. The third integral corresponds to projects with a cost between $\underline{c}(m, N)$ and $\bar{c}(m, N)$; if there are $k \geq m$ high types in the first period, these projects are implemented immediately and 


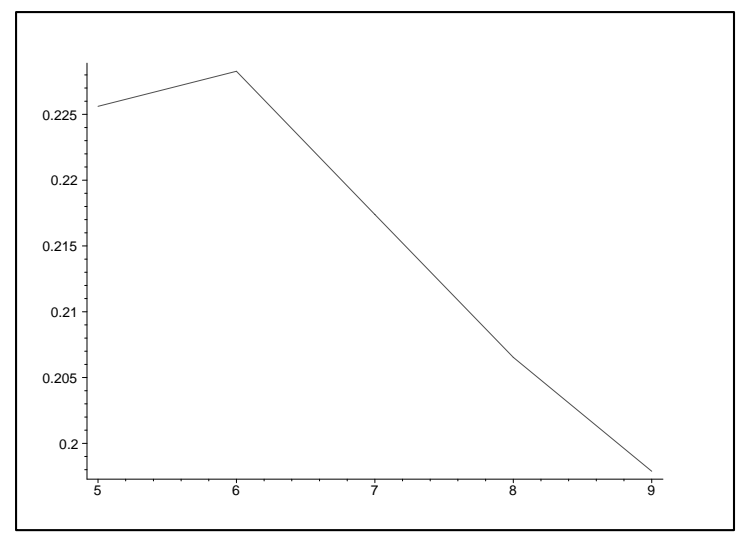

(a) $N=9$

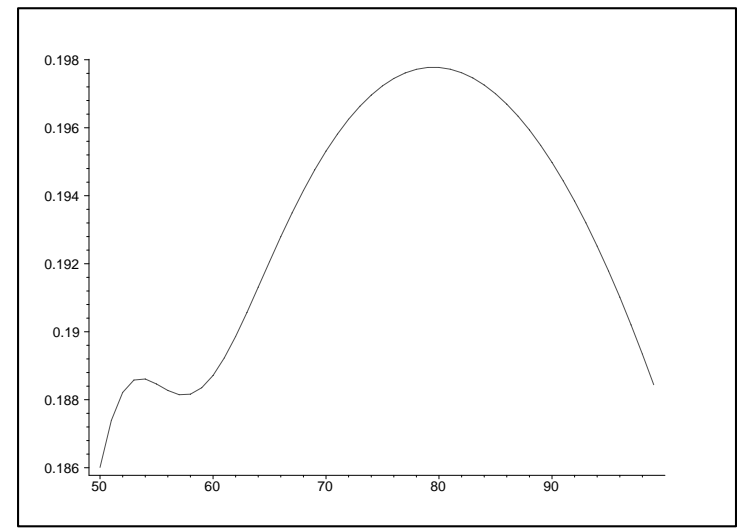

(b) $N=99$

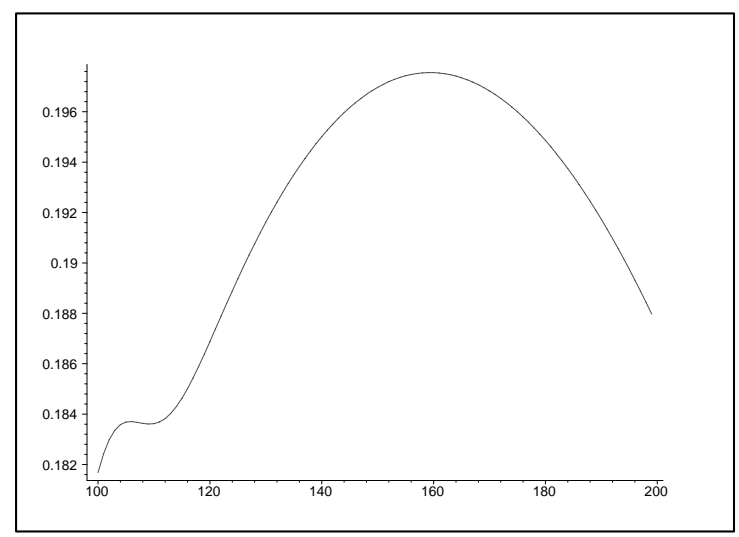

(c) $N=199$

25

Figure 3: Ex-ante expected utility for different majority rules 
generate a per-capita utility of $U_{I}(k, c)$; otherwise, if $k<m$, the project is delayed and each voter obtains the value of waiting.

Figure 3 shows the function $\Pi(m)$ for $N=9, N=99$ and $N=199$. For $N=9$ in part (a), the optimal majority rule is $m=6$, that is, a two-thirds majority rule, just as in the basic model. For $N=99$ in part (b) and $N=199$ in (c), the optimal $m$ is approximately equal to $0.8 N$, respectively. Thus, the optimal majority rule in these cases is considerably larger than in the basic model, where the optimal majority rule is approximately between $7 / 11$ and $2 / 3$.

Intuitively, the reason for why the optimal majority rule increases when voters' second period payoffs are correlated is as follows. Remember that the optimal majority rule in the basic model is (about a 2/3) supermajority rule. If $N$ is at least moderately large, then it is relatively unlikely that a project would be implemented in the second period. This is the reason why low types are willing to agree to first period implementation of projects with a low $c$ : They don't have much to lose in the first period, and potentially a lot to gain in the second period if their second period type is high, but are likely to receive this payoff only if the project is already implemented in the first period. As a consequence, projects with $c \leq \underline{c} \approx 1 / 4$ are implemented unanimously in the first period, and they make up the largest part of all projects that are implemented at all.

Consider what happens when we leave the majority rule unchanged, but second period types are high with probability $\mu$, where $\mu$ is itself a random variable. In this case, it is now much more likely that sufficiently many voters agree in the second period to implement the project. Thus, the value of waiting increases, and first period low types become less inclined to implement a project already in the first period (so $\underline{c}$ decreases). From an ex-ante perspective, the additional projects that are now rejected (i.e., projects with costs between the old and the new level of $\underline{c}$ ) are socially very valuable; since their $c$ is less than $1 / 4$, from a per-capita perspective, they should be implemented based on their first period payoffs for high types alone (the per-capita surplus of these projects is positive even if all voters turn out to be low types in the second period). Thus, the decrease of $\underline{c}$ is very inefficient. Increasing the majority rule reduces the value of waiting, and thus, more projects are accepted (unanimously) in the first period. This effect outweighs the cost that, with a higher majority rule, slightly more (on average efficient) projects are rejected in the second period.

To gain more insight, consider the case of a very large society $(N \rightarrow \infty)$. Let $s=m / N$ denote the proportional majority rule. In the second period, the project is implemented if and only if $\mu>s$. Thus, defining $\mathcal{U}_{W}(c, s)=\lim _{N \rightarrow \infty} U_{W}(c,\lceil s N\rceil, N)$, 
the value of waiting is

$$
\mathcal{U}_{W}(c, s)=\operatorname{Prob}(\mu \geq s)[E(\mu \mid \mu \geq s)-c]=(1-s)\left(\frac{1+s}{2}-c\right) .
$$

It is easy to check that $\mathcal{U}_{W}(c, s)$ is maximal for $s=c$. Intuitively, in the second period a utilitarian social planner would accept a project if and only if the realized percentage of winners $\mu$ is greater than $c$.

When are first period low types just indifferent between implementing the project and waiting? Solving $U_{I}(0, c)=\mathcal{U}_{W}(c, s)$ yields

$$
\underline{c}=\frac{s^{2}}{2(1+s)}
$$

Similarly, first period high types are indifferent between implementing in the first period and waiting if

$$
\bar{c}=\frac{1+\frac{s^{2}}{2}}{1+s}
$$

Under simple majority rule, projects with costs between $\underline{c}$ and $\bar{c}$ are implemented with probability $1 / 2$ in the first period. From (10), we have $\underline{c}=1 / 12$, and from (11), we have $\bar{c}=3 / 4$ under simple majority rule. Thus, the expected ex-ante utility under simple majority rule is

$$
\begin{aligned}
\lim _{N \rightarrow \infty} \Pi\left(\frac{N}{2}, N\right)= & \int_{0}^{1 / 12}(1-2 c) d c+\int_{1 / 12}^{3 / 4}\left[\frac{1}{2}(1-2 c)+\frac{1}{4}\left(\frac{3}{4}-c\right)\right] d c \\
& +\int_{3 / 4}^{1} \frac{1}{2}\left(\frac{3}{4}-c\right) d c=\frac{11}{64}=0.171875 .
\end{aligned}
$$

Consider now a supermajority percentage $s \equiv m / N \geq 0.5+\varepsilon$ (for some $\varepsilon>0$ ). Since the percentage of high types in the first period is almost certainly within $\varepsilon$ of 0.5 , there are almost never sufficiently many high types to implement the project in the first period, if $c>\underline{c}(m, N)$. Thus, the expected ex-ante utility under supermajority rule $s$ is given by

$$
\begin{aligned}
\lim _{N \rightarrow \infty} \Pi(\lceil s N\rceil, N)=\int_{0}^{\underline{c}}(1-2 c) d c+\int_{\underline{c}}^{1}\left(\frac{1+s}{2}-c\right)(1-s) d c \\
=(1-\underline{c})\left[\underline{c}+\frac{1-s^{2}}{2}-\frac{1-s}{2}(1+\underline{c})\right]=\frac{s(2-s)\left(2+2 s-s^{2}\right)}{8(1+s)}
\end{aligned}
$$

where the last line follows from substituting (10). Differentiating (13) with respect to $s$ yields

$$
\frac{3 s^{4}-4 s^{3}-10 s^{2}+4 s+4}{8(1+s)^{2}}
$$


Setting this equal to zero and solving yields that the optimal supermajority rule is approximately $s^{*}=0.7985 .{ }^{19}$ Substituting the optimal value into $\Pi$ yields an expected utility of about 0.1973 , which is larger than the ex-ante utility under simple majority rule. Therefore, a supermajority rule of approximately $80 \%$ is optimal in the limit, which corresponds very well to the maximum in the graphs of Figure 3.

\subsection{Intertemporal correlation}

We now consider the case that the first and second period type of an individual is positively correlated. Specifically, we assume that a voter's second period type coincides with his first period type with probability $r \in[0.5,1]$, i.e. $\operatorname{Prob}\left(V_{2}^{i}=1 \mid V_{1}^{i}=1\right)=$ $\operatorname{Prob}\left(V_{2}^{i}=0 \mid V_{1}^{i}=0\right)=r$. We continue to assume that each player has an equal chance of being a high or a low type in the first period.

Benchmark: No option to wait. As in the basic model, we start by considering the case of a one-off election in period 1 . In our new setting, the net present value of a project with cost parameter $c$ for voter $i$, is

$$
U_{I}\left(V_{1}^{i}, c, r\right)=V_{1}^{i}+E\left[V_{2}^{i} \mid V_{1}^{i}\right]-2 c= \begin{cases}1+r-2 c & \text { if } V_{1}^{i}=1 \\ 1-r-2 c & \text { if } V_{1}^{i}=0\end{cases}
$$

For example, a first period high type gets an immediate payoff of 1 , and is a second period high type with probability $r$. A first period low type gets zero in the first period, and is a second period high type with probability $1-r$.

If the first period decision is final, high types vote in favor of projects with $c \leq$ $(1+r) / 2$, while low types only vote in favor of projects with $c \leq(1-r) / 2$. The stronger is the correlation across periods (i.e., the higher is $r$ ), the more extreme are these cost thresholds, because first period low types have only a very slight hope that they will profit from the project in the second period, while high types are very confident that they will benefit in period 2, too. Essentially, the higher is $r$, the more the social decision problem resembles a situation with known benefits.

The ex-ante expected equilibrium payoff of a project with cost $c$, given $r$ and majority rule $m$, is

$$
\tilde{\pi}(m, c, r)= \begin{cases}1-2 c & \text { if } c \leq \frac{1-r}{2} \\ q(m, N)(1-2 c)+\frac{p(m, N)}{2}(1+r-2 c) & \text { if } \frac{1-r}{2}<c \leq \frac{1+r}{2} \\ 0 & \text { if } c>\frac{1+r}{2}\end{cases}
$$

Just as in the basic model, the ex ante equilibrium payoff is a piecewise linear function of $c$ that exhibits a downward jump at $(1-r) / 2$ and an upward jump at $(1+r) / 2$.

\footnotetext{
${ }^{19}$ The second order condition is satisfied at $s^{*}$.
} 
Following the same arguments as in the proof of Claim 1, we can show that the optimal majority rule for a given $c$ is

$$
m^{*}=\lceil N(2 c-(1-r)) / 2 r\rceil,
$$

which is a decreasing function of $r$. Thus, correlation strengthens the case for low majority requirements, and it is therefore intuitively clear that the result of Proposition 1 also holds here: If project costs are drawn from some distribution that is symmetric around $c=1 / 2$, then simple majority yields the highest average ex ante payoffs if the project decision cannot be postponed.

The option to wait and intertemporal correlation. We now turn to the case that society can implement the project in the second period, if it was turned down in the first period. In order to characterize first period voting behavior, we need to find the value of waiting. In contrast to the basic model, it matters here whether individuals can observe the payoff types of other voters. The number of first period high types influences the distribution of the number of second period high types, and thus the probability of second period implementation. Thus, if voters can observe the first period types of other voters, they will condition their behavior on it. If, instead, types are only privately observed, then each voter has to take into account the first period type distribution conditional on the event that his vote is decisive in the first period election.

In what follows, we assume that payoff types are publicly observed. We make this assumption for two reasons. First, this assumption is probably reasonable for applications with small electorates. Second, a model with publicly observed types is more tractable than a model with privately observed payoff types. In particular, with publicly observable payoff types, iterated elimination of weakly dominated strategies still delivers a unique strategy profile (up to tie breaking in situations where individuals are indifferent between their two first period actions, independently of other voters' behavior).

The same is not true for a model with privately observed types, where the voting game may exhibit multiple (sequential) equilibria in iteratively weakly undominated strategies. A sufficiently detailed exposition of such a game would considerably increase the length of the paper. Moreover, in qualitative terms, the interesting results do not change substantially from the case considered here. ${ }^{20}$

We start our analysis by defining voter $i$ 's value of waiting, which now depends not only on the cost parameter $c$ and the majority rule $m$, but also on the intertemporal

\footnotetext{
${ }^{20} \mathrm{~A}$ formal analysis of the voting game with privately observed types is available from the authors upon request.
} 
correlation parameter $r$, voter $i$ 's first period type and the number of high first period types among other voters, $h$, which determines the distribution of the number of second period high types and hence the probability of second period implementation. Formally, we have

$$
\begin{gathered}
U_{W}\left(V_{1}^{i}, c, m, h, r\right)=E\left[\max \left\{V_{2}^{i}-c, 0\right\} \mid V_{1}^{i}\right] p_{2}(m, h, r)+\left(E\left[V_{2}^{i} \mid V_{1}^{i}\right]-c\right) q_{2}(m, h, r) \\
= \begin{cases}(1-c)(1-r) p_{2}(m, h, r)+(1-r-c) q_{2}(m, h, r) & \text { if } V_{1}^{i}=1 \\
(1-c) r p_{2}(m, h, r)+(r-c) q_{2}(m, h, r) & \text { if } V_{1}^{i}=0 .\end{cases}
\end{gathered}
$$

The functions $p_{2}$ and $q_{2}$ are generalizations of the corresponding functions in the basic model and represent the probability of being pivotal in period two, and the probability that the project will pass in period two independently of voter $i$ 's will, respectively. In order to formally define $p_{2}$ and $q_{2}$, consider the transition function

$$
t(\ell, k, r)=\sum_{j=0}^{k}\left(\begin{array}{l}
\ell \\
j
\end{array}\right)\left(\begin{array}{c}
N-1-\ell \\
k-j
\end{array}\right)(1-r)^{k+l-2 j} r^{N-\ell-1-k+2 j},
$$

which describes the probability of moving from a first period type profile in which $l$ of the $N-1$ other players have high types to a second period profile in which $k$ of them have high types. The functions $p_{2}$ and $q_{2}$ can now be written as

$$
p_{2}(m, h, r)=t(h, m-1, r) \quad \text { and } \quad q_{2}(m, h, r)=\sum_{j=m}^{N-1} t(h, j, r) .
$$

Just as in the basic model, both (14) and (16) are linearly decreasing functions of $c$. Moreover, (14) decreases faster than (16). It is also obvious that, for $c$ sufficiently close to 0 , implementing the project immediately is strictly better than waiting, irrespective of the values of $V_{1}^{i}, h, m$ and $r$. Similarly, if $c$ is sufficiently close to 1 , then delaying the project dominates investing immediately for all parameter values. Thus, for each tuple $(h, m, r)$, there are cost thresholds $\underline{c}(h, m, r)$ and $\bar{c}(h, m, r)$ at which high and low types switch from approval to rejection, respectively. Since

$U_{W}(0,(1-r) / 2, m, h, r)=\frac{(1-r)}{2}\left((1+r) p_{2}(m, h, r)+q_{2}(m, h, r)\right)>0=U_{I}(0,(1-r) / 2, r)$,

it follows that $\underline{c}(m, h, r)<(1-r) / 2$ for all $(m, h, r)$. Similarly, for high types we have

$$
U_{W}(1, m, r, h, r)=(1-r) r p_{2}(m, l, r)<1-r=U_{I}(1, r, r)
$$

which implies that $\bar{c}(m, h, r)>r$ for all $(m, h, r)$. Results that parallel Proposition 3 for the behavior of $\underline{c}$ and $\bar{c}$ can be obtained, but we refrain from discussing them explicitly in order to save some trees. 
We now turn to the definition of the ex-ante expected equilibrium payoff. It is convenient to calculate this expectation in two steps: First, we integrate over a player's payoff type, given the number of other players who are high types, $h$. Let $C_{m, h, r}^{0}=\{c \mid c \leq$ $\underline{c}(m, h, r)\}, C_{m, h, r}^{1}=\{c \mid c \in(\underline{c}(m, h, r), \bar{c}(m, h, r)]\}$, and $C_{m, h, r}^{2}=\{c \mid c>\bar{c}(m, h, r)\}$. For any triple $(c, m, r)$, voter $i$ 's expected payoff, conditional on there being $h$ high types among the other voters, is

$$
\pi_{h}(c, m, r)= \begin{cases}\frac{U_{I}(1, c, r)+U_{I}(0, c, r)}{2} & \text { if } c \in C_{m, h, r}^{0} \vee\left(c \in C_{m, h, r}^{1} \wedge h \geq m\right) \\ \frac{U_{I}(1, c, r)+U_{W}(0, m, c, h, r)}{2} & \text { if } c \in C_{m, h, r}^{1} \wedge h=m-1 \\ \frac{U_{W}(1, m, c, h, r)+U_{W}(0, m, c, h, r)}{2} & \text { if } c \in C_{m, h, r}^{2} \vee\left(c \in C_{m, h, r}^{2} \wedge h<m-1\right)\end{cases}
$$

Second, we will now take the expectation with respect to $h$, which gives

$$
\pi(c, m, r)=\frac{1}{2^{N-1}} \sum_{h=0}^{N-1}\left(\begin{array}{c}
N-1 \\
h
\end{array}\right) \pi_{h}(c, m, r) .
$$

As in the basic model, this function a piecewise linear function of $c$. Of course, $\pi(c, m, r)$ exhibits more than just two discontinuities, since both high and low type voters have multiple thresholds (one for each $h$ ) at which behavior switches. Figure 4 shows for the case that $r=2 / 3$, the expected payoffs for $N=15$ under the two majority rules $m=8$ and $m=9$.

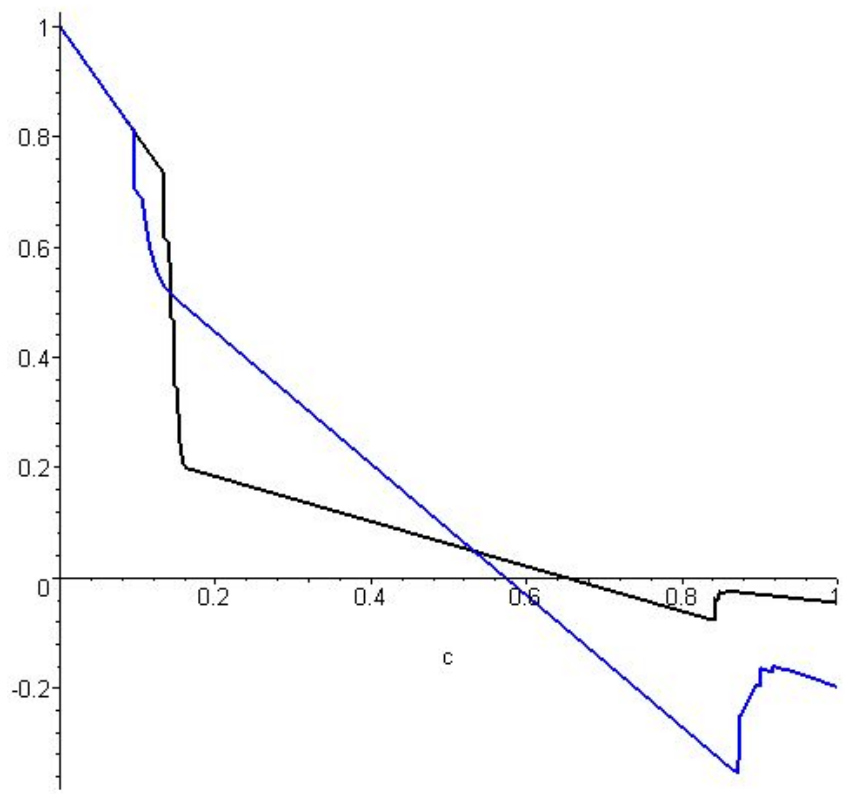

Figure 4: Ex-ante average payoff: $N=15, m=8$ (blue) and $m=9$ (black) 
One of the central results of the basic model was that, once the option to wait is introduced, simple majority is dominated by all supermajority rules. In comparison to the basic model, intertemporal correlation strengthens the case for simple majority rule. This is quite clear in the case of $r=1$, because the dynamic structure of our model then becomes irrelevant: If individuals' benefits are constant over time, then the set of voters who approve remains constant, and hence, a project is either implemented at once, or not at all. This is exactly the same behavior as in the benchmark model without the option to wait, where we know that simple majority maximizes the ex-ante average payoffs.

It is intuitive that, as we increase $r$ from $1 / 2$ (i.e., the basic model) to $r=1$ (i.e., perfect correlation), the optimal supermajority rule decreases. It is an interesting quantitative question to consider for which levels of $r$ the optimal supermajority rule switches.

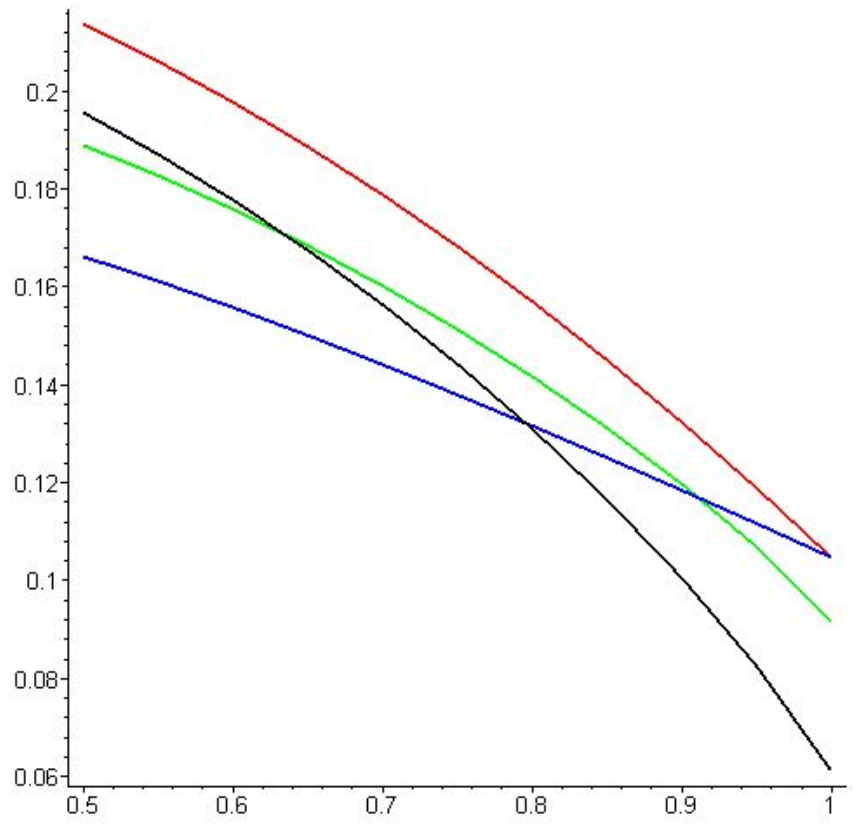

Figure 5: Ex-ante average payoff for $N=15: m=8$ (blue), $m=9$ (green), $m=10$ (black); and for $m=8$, without the option of waiting (red)

Figure 5 shows, for $N=15$, the average ex-ante payoff $\Pi(m, r)=\int_{0}^{1} \pi(c, m, r)$ as a function of $r$ for different majority rules. Consider first the three lower curves that intersect each other. The blue curve in Figure 5, the flattest of the three, represents the payoffs under simple majority, i.e. $m=8$, while the green and the black one (the steepest one) show the payoffs under the supermajority rules $m=9$ and $m=$ 10, respectively. Note that $m=10$, i.e., a two-thirds majority rule, is the optimal 
majority rule in the basic model. As can be seen, simple majority is dominated by both supermajority rules for any persistence level up to roughly $r=0.8$. Moreover, the majority rule $m=9$ (i.e. a $60 \%$ rule) continues to outperform simple majority for all $r$ from 0.8 through (about) 0.92. Thus, even for a very strong level of intertemporal correlation of payoffs, a supermajority rule remains optimal. This example therefore suggests that, while intertemporal correlation decreases the optimal majority rule, our qualitative result concerning the optimality of supermajority rules is very robust even at high levels of correlation. ${ }^{21}$

The last, red, curve shows the ex-ante expected payoff without the option of waiting, that is, when society has to implement the project either immediately or not at all. From our discussion in the basic model, we know already that, for $r=0.5$ this payoff is higher than the payoff with the option to wait, even under the optimal supermajority rule. Figure 4 shows that this result continues to hold for $r>1 / 2$, except at $r=1$ where the difference between the payoffs with and without the option to wait becomes zero. This is intuitive, as with perfect correlation, a project is either implemented immediately or not at all, so payoffs are the same, whether or not second period implementation is, in principle, possible.

\section{Previous literature on supermajority rules}

Our results shed light on an important question in the endogenous determination of institutions: Why do some organizations choose supermajority rules, and which features of decision problems influence this choice? In this section, we relate our model to previous literature on this subject.

Majority rules within organizations vary considerably, from simple majority rule to unanimity rule. Often, the choice of the majority rule that is to govern future decision making is a contentious issue itself, such as in the recent EU summit, which, in the end, adopted some form of a supermajority rule. Supermajority rules are also used in most countries for a change of the constitution, and, often implicitly, for "normal" legislation. For example, in parliamentary systems with a strong committee organization, a legislative proposal usually needs the support of both the respective committee and the house. In parliamentary systems with two chambers, certain legislative proposals need the support of both chambers. ${ }^{22}$

\footnotetext{
${ }^{21}$ This result is further strengthened by the fact that $N=15$ is relatively small in our example, so that even a simple majority rule requires the approval of $8 / 15=53.3 \%$ of the population, and the smallest possible supermajority rule is already a $60 \%$ rule.

${ }^{22}$ Tullock (1998), p.216, estimates that legislative rules in the US for changing the status quo are "roughly equivalent to requiring a $60 \%$ majority in a single house elected by proportional representation." See also Diermeier and Myerson (1999).
} 
Several previous papers have analyzed arguments for supermajority rules from an economic point of view. Buchanan and Tullock (1962) argue for unanimity rule as the suitable rule governing social choices. Under a simple majority rule, a majority of people could be tempted to implement certain projects that are not socially desirable because they can "externalize" part of the cost associated with this project to the losing minority. Under unanimity rule, only Pareto improving projects are implemented. However, Guttman (1998) has argued that the unanimity rule leads to a rejection of many projects that are not Pareto improvements, but nevertheless worthwhile from a reasonable social point of view. Assuming that the social goal is to minimize the sum of both types of mistakes, he shows that simple majority rule is optimal. Our model is constructed in a way that simple majority rule would also be optimal if voters have to make a once-and-for-all decision about the project in the first period. However, with the option to postpone a decision to the second period, we show that (in the same symmetric setting), a supermajority rule is optimal.

Another rationale for supermajority rules is that this counteracts the problem of time inconsistency of optimal policies (see, e.g., Gradstein (1999) or Dal Bo (2006)). For example, a constitution that protects investment by inhibiting nationalization is valuable only if the constitution cannot be easily changed after investment has taken place. In our model, time inconsistency is not an issue.

As is well known, simple majority rule may lead to cycles in electoral preferences. A higher required majority reduces the possibility of cycles. Indeed, Caplin and Nalebuff (1988) show that a $\left(1-(n /(n+1))^{n}\right)$ supermajority rules out cycles, if voters have single peaked Euclidean preferences in an $n$-dimensional space. In our model, the decision is binary in each period, so cycles never arise in our model.

Aghion and Bolton (2003) analyze the optimal choice of the majority rules in a model where a polity has to decide simultaneously about public good provision and costly redistribution, and redistribution creates a deadweight loss. They assume that the constitutional rules are written before voters learn the costs and benefits of the public good. The optimal majority rule trades off the higher ex-post flexibility of low majority rules (which lead to more efficient public good provision) against the protection against excessive redistribution afforded by supermajority rules.

Messner and Polborn (2004) analyze an overlapping generations model in which voters know that their preferences over reform projects will become more conservative over the remainder of their lives. The initial population decides on the majority rule to be used for later decisions. The median voter in the constitutional election prefers to implement a supermajority rule, which allows him to transfer power to his (more conservative) "average future self". In contrast to Messner and Polborn (2004), the electorate remains constant over time in our model, thus removing the incentive for 
the initial generation to use supermajority rules in order to transfer power from future voters to themselves. Also, the implementation decision on any reform project in Messner and Polborn (2004) is a simple one-time, up-or-down vote, while our focus here is on the timing of the implementation of reforms.

The implications of different majority rules have also been analyzed in settings where voters have congruent interests, but are only imperfectly informed about the consequences of the different alternatives. Inspired by Condorcet's famous Jury Theorem, ${ }^{23}$ several authors have analyzed which majority rule is most efficient in aggregating the information that is dispersed in the electorate. Nitzan and Paroush (1985) find that the probability of a correct choice is maximized under simple majority rule. Feddersen and Pesendorfer (1998) analyze information aggregation with strategic voters and show that simple majority rule is optimal for information aggregation purposes, while unanimity rule is dominated by all other majority rules, if there are sufficiently many voters. Bond and Eraslan (2007) show that supermajority or unanimity rules may have an advantage for voters even in an information aggregation setting, if the proposal is made by an agenda setter whose interest is diametrically opposed to the voters. The intuition is that unanimity rule forces the agenda setter to make a more favorable proposal to voters, and this outweighs the disadvantage that, with positive probability, voters make mistakes under unanimity rule. The issue of information aggregation is not present in our model as all voters hold, at all times, the same information.

\section{Discussion and conclusions}

We analyze a model in which voters have to choose whether to implement a project immediately, or wait till the second period and reconsider the decision then. Our main focus is the interplay of individual learning and social decisions, and how this is influenced by the majority rule that governs the decision making process. We characterize whether individual voting behavior becomes more or less conservative when the majority rule is increased, and we show how this is related to the effect of the majority rule on the value of waiting.

Our main conclusions were twofold: First, we show that a supermajority rule dominates simple majority rule with respect to social welfare. We have shown that the optimal supermajority rule is larger than $7 / 11 \approx 63.6$ percent, and, for a large society, also not larger than a two-thirds majority rule. Correlation between first and second period valuations reduces the size of the optimal majority rule. Perfect correlation es-

\footnotetext{
${ }^{23}$ Condorcet's Jury Theorem states that under simple majority rule, the probability with which a society facing a binary choice problem makes the correct choice, converges to one as the number of voters increases.
} 
tablishes simple majority rule as the optimal rule; however, even if correlation is high, but not perfect, the optimal majority rule remains a supermajority rule.

Our second main result was that the option of waiting, which is always positive for individual decision problems, can be negative for our social decision problem. Indeed, we show that this is the case when the project cost is uniformly distributed from an ex-ante perspective, even if society adopts the optimal majority rule in the case that they have the option to wait.

Our model provides a fundamentally new rationale for societies choosing supermajority rules. Our principal effect in our model relies on voters' uncertainty over the consequences of project implementation, and the option value of waiting until new information is available. Thus, our model is most relevant for societies that frequently face decision problems with such characteristics.

For example, one can argue that the European Union fits this description quite well. The most important decisions that are made in the EU framework concern the admission of new members, transnational investment projects like the introduction of the Euro and the harmonization of industry regulations. One can argue that many of these projects are less "standard" (relative to the most important policy issues in the member states) and have uncertain payoff consequences for the member states. Interestingly, the European Council (the council of member state governments that makes the most significant decisions) uses a supermajority rule.

Also, most countries require supermajorities for changes of their constitution. Again, this area appears closer to the setting of this model than ordinary legislation issues: At the time when the constitution is written, future needs are difficult to foresee and potential winners and losers are unclear, and even once a proposal arises, the consequences of changes for the distribution of gains and losses are not necessarily clear.

In contrast, most ordinary legislation in national legislatures concerns social or economic issues where preferences are more stable and well-known. As we have seen in Section 4.2, the higher the correlation of voter types over time (and therefore, the smaller the opportunity of learning), the closer is the ex-ante optimal majority rule to simple majority rule.

After all the astonishing results we have shown, the reader may have concluded that we have succeeded in getting very close to proving the world formula. Intellectual honesty, though, requires us to admit that, while this paper indeed constitutes an important step in the right direction, there is still some way to go.

One direction in which future research can expand on our model framework is as follows. In our model, individuals only choose how to vote. In some instances, individuals may also be able to adapt to the policy enacted and thereby influence the distribution of their payoff in the second period. This may be important, for example, 
in issues where the project is some sort of environmental regulation, say, increasing the private cost of some polluting activity. Adaptation (say, buying a smaller car, isolating one's home) may make compliance less costly over time, but the enacted policy (as well as the expectation of which regulation will be in force in the next period) will affect the optimal extent to which individuals adapt. 


\section{Appendix}

Proof of Lemma 1. Consider the difference $U_{W}(c, m+1)-U_{W}(c, m)=$

$$
\begin{aligned}
& \left(\frac{1}{2}-c\right)(q(m+1, N)-q(m, N))+\frac{1-c}{2}(p(m+1, N)-p(m, N)) \\
= & \frac{1-c}{2}[p(m+1, N)-p(m, N)]-\left(\frac{1}{2}-c\right) p(m+1, N),
\end{aligned}
$$

where the second line results from substituting the identity $q(m, N)=p(m+1, N)+$ $q(m+1, N)$. The expression in (17) is positive if and only if

$$
c \geq \frac{p(m, N)}{p(m, N)+p(m+1, N)}=\frac{\left(\begin{array}{c}
N-1 \\
m-1
\end{array}\right)}{\left[\left(\begin{array}{c}
N-1 \\
m-1
\end{array}\right)+\left(\begin{array}{c}
N-1 \\
m
\end{array}\right)\right]}=\frac{\left(\begin{array}{c}
N-1 \\
m-1
\end{array}\right)}{\left(\begin{array}{c}
N \\
m
\end{array}\right)}=\frac{m}{N} .
$$

Lemma 4. For all $N$ and $(N+1) / 2 \leq m \leq N, \frac{q(m, N)}{p(m, N)} \leq \frac{N-m}{2 m-N}$.

Proof. Fix $N$ and $m$ and let $s=m / N$. Observe that

$$
\begin{aligned}
\frac{q(m, N)}{p(m, N)} & =\sum_{\ell=m+1}^{N} \frac{p(\ell, N)}{p(m, N)}=\frac{\sum_{\ell=m}^{N-1}\left(\begin{array}{c}
N-1 \\
\ell
\end{array}\right)}{\left(\begin{array}{l}
N-1 \\
m-1
\end{array}\right)}=\sum_{\ell=m}^{N-1} \frac{(m-1) !(N-m) !}{\ell !(N-1-\ell) !} \\
& =\sum_{\ell=m}^{N-1} \frac{(N-\ell) \cdots(N-m)}{m \cdots \ell}=\sum_{\ell=m}^{N-1} \prod_{k=m}^{\ell} \frac{N-k}{k} .
\end{aligned}
$$

Since $(N-k) / k \leq(N-m) / m=(1-s) / s$ for all $k \geq m$ it follows that the right hand side of (18) is smaller than

$$
\begin{gathered}
\sum_{\ell=m}^{N-1}\left(\frac{1-s}{s}\right)^{\ell-m+1}=\sum_{\ell=0}^{N-m-1}\left(\frac{1-s}{s}\right)^{\ell+1} \leq \\
\frac{1-s}{s} \sum_{\ell=0}^{\infty}\left(\frac{1-s}{s}\right)^{\ell}=\frac{1-s}{2 s-1}=\frac{N-m}{2 m-N} .
\end{gathered}
$$

Proof of Lemma 2. Observe that $\frac{p(m+1, N)}{q(m+1, N)} \geq \frac{p(m, N)}{q(m, N)}$ if and only if

$$
\frac{p(m, N)}{p(m+1, N)} \leq \frac{q(m, N)}{q(m+1, N)}=\frac{q(m+1, N)+p(m+1, N)}{q(m+1, N)}=\frac{p(m+1, N)}{q(m+1)}+1
$$

Rearranging and using $p(m, N) / p(m+1, N)=m /(N-m)$, this becomes

$$
\frac{N-m}{2 m-N} \geq \frac{q(m+1, N)}{p(m+1, N)} .
$$

By Lemma 4 in the Appendix, we have that $\frac{q(m+1, N)}{p(m+1, N)} \leq \frac{N-(m+1)}{2(m+1)-N}$. Thus, since $\frac{N-m}{2 m-N}>\frac{N-(m+1)}{2(m+1)-N},(20)$ is always satisfied, so that $\frac{p(m, M)}{q(m, N)}$ is increasing in $m$. 
Proof of Lemma 3. Observe that $U_{W}(c, m)=(1-c) p(m, N) / 2+(1 / 2-c) q(m, N)$ is negative if and only if $c>[p(m, N)+q(m, N)] /[p(m, N)+2 q(m, N)]$. This, in turn, is equivalent to

$$
c>1-\frac{q(m, N)}{p(m, N)+2 q(m, N)}=1-\frac{1}{\frac{p(m, N)}{q(m, N)}+2} .
$$

Thus, if, for a given $c$, this inequality is satisfied at some majority rule $m$, then it must also be satisfied for any $m^{\prime}<m$, because the denominator on the right hand side is increasing in $m$ by Lemma 2 .

Lemma 5. $N p(\lceil 3 N / 4\rceil, N) \leq 4$ for all $N$.

Proof. Given that $N$ is an odd number we have that either $3 N+1$ is divisible by 4 (for $N=5,9,13, \ldots$ ) or $3(N+1) / 4$ is an integer (for $N=3,7,11, \ldots)$. In the first case we have that $\lceil 3 N / 4\rceil=(3 N+1) / 4$, while in the latter case we have $\lceil 3 N / 4\rceil=3(N+1) / 4$. Notice also that in either case we have $\lceil 3(N+4) / 4\rceil-\lceil 3 N / 4\rceil=3$.

Let $f(N)=N p(\lceil 3 N / 4\rceil, N)$. In what follows we will show that $f(N+4)-f(N)<$

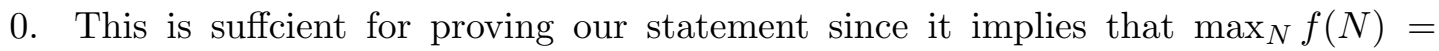
$\max \{f(3), f(5)\}=\max \left\{3\left(\begin{array}{l}2 \\ 2\end{array}\right) / 2^{2}, 5\left(\begin{array}{l}4 \\ 3\end{array}\right) / 2^{4}\right\}=5 / 4<4$.

Letting $m=\lceil 3 N / 4\rceil$ the increment $f(N+4)-f(N)$ is given by

$\frac{N+4}{2^{N+3}}\left(\begin{array}{l}N+3 \\ m+2\end{array}\right)-\frac{N}{2^{N-1}}\left(\begin{array}{l}N-1 \\ m-1\end{array}\right)=\frac{N}{2^{N-1}}\left(\begin{array}{l}N-1 \\ m-1\end{array}\right)\left[\frac{(N+4)(N+3)(N+2)(N+1)}{16(N+1-m)(m+2)(m+1) m}-1\right]$.

Observe that $(N+j+2)<4(m+j)$ for $j=0,1,2$ and that $N+1 \leq 4(N+1-m)$. Thus the first term in the square brackets is a product of four numbers which are smaller than one, and thus the term in square brackets is negative.

Proof of Proposition 3, part 2. Remember that the threshold $\bar{c}(m)$ solves the equation

$$
\frac{3}{2}-2 c=\frac{1-c}{2} p(m, N)+\left(\frac{1}{2}-c\right) q(m, N) .
$$

Thus, we have

$$
\bar{c}(m)=\frac{3-q(m, N)-p(m, N)}{4-2 q(m, N)-p(m, N)} .
$$

Dropping the variables from the functions $p$ and $q$ and denoting their values at $m+1$ by $p^{\prime}$ and $q^{\prime}$ respectively we have

$$
\bar{c}(m) \geq \bar{c}(m+1) \quad \Leftrightarrow \quad \frac{4-2 q-p}{3-q-p}=1+\frac{1-q}{3-q-p} \leq 1+\frac{1-q^{\prime}}{3-q^{\prime}-p^{\prime}}=\frac{4-2 q^{\prime}-p^{\prime}}{3-q^{\prime}-p^{\prime}}
$$


which may be written equivalently as

$$
\frac{3-q-p}{1-q}=1+\frac{2-p}{1-q} \geq 1+\frac{2-p^{\prime}}{1-q^{\prime}}=\frac{3-q^{\prime}-p^{\prime}}{1-q^{\prime}} .
$$

Using $q^{\prime}=q-p^{\prime}$ and $p^{\prime} / p=(N-m) / m$ we can express this condition as follows

$$
\begin{aligned}
(2-p)\left(1-q+p^{\prime}\right)-\left(2-p^{\prime}\right)(1-q) & =p\left(\frac{3 p^{\prime}}{p}-1+q-\frac{p^{\prime}}{p} p+\frac{p^{\prime}}{p} q\right) \\
& =p\left(\frac{3 N-4 m}{m}+q \frac{N}{m}-\frac{N-m}{m} p\right) \geq 0 .
\end{aligned}
$$

Hence, it follows that $\bar{c}$ is decreasing at $m$ if and only if

$$
3 N-4 m+N q-(N-m) p \geq 0 .
$$

Now observe that, since $q>p^{\prime}$ and $p^{\prime} / p=(N-m) / m$, we have that

$N q-(N-m) p>N p^{\prime}-(N-m) p=N p \frac{p^{\prime}}{p}-(N-m) p=(N-m) p\left(\frac{N}{m}-1\right) \geq 0$.

Since $3 N-4 m>0$ for all $m<3 N / 4$, we can therefore conclude that $\bar{c}$ must be decreasing at least up to $m=\lfloor 3 N / 4\rfloor$.

Next we argue that if (22) is satisfied at $m<N$ then it must also hold at $m^{\prime}=m+1$. To see this, evaluate (22) at $m$ and at $m+1$, and observe that the difference is

$$
\begin{array}{r}
3 N-4 m+N q-(N-m) p-\left[3 N-4(m+1)+N q^{\prime}-(N-m-1) p^{\prime}\right]= \\
4+p^{\prime}[2(N-m)-1]>0 .
\end{array}
$$

It follows that $\bar{c}$ is increasing from $m=\lceil 3 N / 4\rceil$ onwards if it is so at $m=\lceil 3 N / 4\rceil$. Notice that at $m=\lceil 3 N / 4\rceil$ we have $4 m-3 N \geq 1$ and so we only have to show that $N q(\lceil 3 N / 4\rceil, N)-(N-\lceil 3 N / 4\rceil) p(\lceil 3 N / 4\rceil, N)<1$.

From Lemma 4 , we know that $p \geq q(2 m-N) /(N-m)$, and thus

$$
\begin{aligned}
& N q(\lceil 3 N / 4\rceil, N)-(N-\lceil 3 N / 4\rceil) p(\lceil 3 N / 4\rceil, N) \\
& \quad \leq 2(N-\lceil 3 N / 4\rceil) q(\lceil 3 N / 4\rceil, N) \leq \frac{N}{2} q(\lceil 3 N / 4\rceil, N) \leq \frac{N}{4} p(\lceil 3 N / 4\rceil, N)<1 .
\end{aligned}
$$

where Lemma 4 is used both for the first and the third inequality, and the last inequality follows from Lemma 5 .

Finally, observe that since $q((N+1) / 2, N)+p((N+1) / 2, N) / 2=1 / 2, p((N+$ 1) $/ 2, N) \leq 1 / 2$ and $q(N, N)=0$ we have

$$
\begin{aligned}
\bar{c}((N+1) / 2) & =\frac{5-p((N+1) / 2, N)}{6} \geq \frac{3}{4} \text { and } \\
\bar{c}(N) & =\frac{3-p(N, N)}{4-p(N, N)}<\frac{3}{4} .
\end{aligned}
$$


Proof of Proposition 5. In the interest of a compact notation in what follows we write $m^{\prime}=m+1, q^{\prime}=q\left(m^{\prime}, N\right)$ and $p^{\prime}=p\left(m^{\prime}, N\right)$; the probabilities which refer to the majority rule $m$ instead are simply denoted by $p$ and $q$, respectively.

We have to show that the difference $\Pi\left(m^{\prime}\right)-\Pi(m)$ is negative whenever $m \geq 2 N / 3$. It is a matter of tedious but straightforward algebraic manipulations that this difference is equal to the ratio ${ }^{24}$

$$
\frac{2 p q p^{\prime}+8 q p-11 p-8 p p^{\prime}-q^{2} p-p\left(p^{\prime}\right)^{2}-16 q p^{\prime}+21 p^{\prime}-2 q\left(p^{\prime}\right)^{2}+4\left(p^{\prime}\right)^{2}+3 q^{2} p^{\prime}}{4\left(4-2 q+p^{\prime}\right)(4-2 q-p)} .
$$

The denominator of this expression is clearly positive. Thus the sign of the difference in average ex-ante payoffs coincides with the sign of the numerator of this expression. Denote this numerator by $d\left(p, q, p^{\prime}\right)$.

We first show that for any $p$ and $q, d$ is monotonically increasing in $p^{\prime}$. We have

$$
\begin{aligned}
\frac{\partial d\left(p, q, p^{\prime}\right)}{\partial p^{\prime}} & =2 p q-8 p-2 p p^{\prime}-16 q+21-4 q p^{\prime}+8 p^{\prime}+3 q^{2} \\
& \geq-8 p-2 p p^{\prime}-16 q+21-4 q p^{\prime} \geq 13-2 p p^{\prime}-4 q p^{\prime}>0,
\end{aligned}
$$

where the second inequality sign in this expression follows from the fact that $q+p / 2 \leq$ $1 / 2$.

Since $p^{\prime}=(N-m) p / m$, we have that $p^{\prime} \leq p / 2$ for all $m \geq 2 N / 3$, and thus

$d\left(p, q, p^{\prime}\right) \leq \max _{p^{\prime} \leq p / 2} d\left(p, q, p^{\prime}\right)=d(p, q, p / 2)=p\left[\frac{p q}{2}-\frac{1}{2}-3 p+\frac{q^{2}}{2}-\frac{p^{2}}{4}\right]<\frac{p}{2}\left[p q-1+q^{2}\right]$.

Given that $p, q \leq 1 / 2$ we thus have that $d\left(p, q, p^{\prime}\right)<0$ whenever $m \geq 2 N / 3$. This proves the first part.

Since $p^{\prime}=(N-m) p / m=\frac{1-s}{s} p$ for $s=m / N$, we have $p^{\prime} \geq a p$ for $m \leq s N$, where $a=(1-s) / s$. Monotonicity of $d$ in $p^{\prime}$ therefore implies that

$$
\begin{aligned}
d\left(p, q, p^{\prime}\right) & \geq \min _{p^{\prime} \geq a p} d\left(p, q, p^{\prime}\right)=d(p, q, a p) \\
& =\{[a(4-p)-8] a p+[2 p a(1-a)+(3 a-1) q-8(2 a-1)] q+21 a-11\} p .
\end{aligned}
$$

Now observe that the sign of $\partial d(p, q, a p) / \partial q$ coincides with the sign of $2 p a(1-a)+$ $(6 a-2) q-8(2 a-1)$. Since

$$
2 p a(1-a)+(6 a-2) q-8(2 a-1)<8-a(16-2(2 q+p))+2 q(1-a) \leq 8-14 a
$$

it follows that whenever $a \geq 8 / 14=4 / 7$ then $\min _{p^{\prime} \geq a p} d\left(p, q, p^{\prime}\right)$ is decreasing in $q$. Using the fact that $q \leq(1-p) / 2$ we thus have that for all $1 \geq a \geq 4 / 7$

$$
\begin{aligned}
d\left(p, q, p^{\prime}\right) & \geq \min _{q \leq(1-p) / 2}\left\{\min _{p^{\prime} \geq a p} d\left(p, q, p^{\prime}\right)\right\}=d(p,(1-p) / 2, a p) \\
& =\frac{p}{4}\left(55 a-29-2 a p-a p^{2}-14 p+12 a^{2} p-p^{2}\right)=: D(p, a) .
\end{aligned}
$$

\footnotetext{
${ }^{24}$ The following expression is obtained by using $q^{\prime}=q-p^{\prime}$.
} 
$D(p, a)$ is clearly increasing in $a$. In the case of simple majority we have $a=(N-$ $1) /(N+1)$, which is increasing in $N$. Thus under simple majority we have that $a \geq 2 / 3$ if $N \geq 5$ (for $N=3$ all majority rules satisfy $m \geq 2 N / 3$ ). Since $D(p, 2 / 3)=p(23-$ $\left.30 p-5 p^{2}\right) / 12>0$ for all $p \in(0,1 / 2)$ we can therefore conclude that at simple majority the increment of $\Pi$ is positive.

The preceding observations allow us to restrict our attention in the remainder of the proof to supermajority rules $m>(N+1) / 2$. Since for all such rules we have $1 / 2 \geq p(m-1, N) / 2+q(m-1)$ and $p(m, N) \geq p(m-1, N) / 2$, the identity $q(m-1, N)=$ $p(m, N)+q(m, N)$ implies $3 p(m, N) / 2+q(m, N) \leq 1 / 2$ or equivalently $q \leq(1-3 p) / 2$. Exploiting this fact we can thus claim that if $m>(N+1) / 2$ then we have for all $a \in(4 / 7,1)$ that

$$
\begin{aligned}
d\left(p, q, p^{\prime}\right) & \geq \min _{q \leq(1-3 p) / 2}\left\{\min _{p^{\prime} \geq a p} d\left(p, q, p^{\prime}\right)\right\}=d(p,(1-3 p) / 2, a p) \\
& =\frac{p}{4}\left(50 a p+15 p^{2} a-29-42 p-9 p^{2}+8 a^{2} p^{2}+55 a+12 a^{2} p\right)=: \hat{D}(p, a) .
\end{aligned}
$$

Since $\hat{D}(p, a)$ is strictly increasing in $a$ it follows that for all $a \geq 4 / 7$ we have

$$
d\left(p, q, p^{\prime}\right) \geq \hat{D}(p, 4 / 7)=\frac{p\left(119-466 p+107 p^{2}\right)}{196} .
$$

It is straightforward to see that this expression is strictly positve for all $p \in(0,1 / 4]$. Since $p(m, N)$ is decreasing in $m$ for every $N$ and also $p((N+3) / 2, N)$ decreases with $N$, it follows that for $2 N / 3>m>(N+1) / 2$ we must have $p(m, N) \leq p(7,11)=$ $105 / 520<1 / 4$ (notice that only for $N \geq 11$ there are majority rules in the specified range). Hence, for all $p$ which may arise for $2 N / 3>m>(N+1) / 2$ we know that $\hat{D}(p, a)>0$, whenever $a \geq 4 / 7$. The condition $a=(1-s) / s \geq 4 / 7$ in turn is equivalent to $s=m / N \leq 7 / 11 \approx 0.636$. Thus we can conclude that $d\left(p, q, p^{\prime}\right)>0$ for all $(N+1) / 2<m<7 N / 11$. This proves statement ii).

Finally, using $p((N+1) / 2, N) / 2+q((N+1) / 2, N)=1 / 2$ and $q(N, N)=0$ it is straightforward to show that

$$
\Pi(N)-\Pi((N+1) / 2)=\frac{16-27 p+10 p^{2}+p^{3}}{48(4-p)},
$$

which is strictly positive for all $p<1$.

Proof of Proposition 6. Like in earlier proofs, we drop the arguments the functions $p$ and $q$. Calculating the difference between $\Pi(m, N)$ and $\tilde{\Pi}(m, N)$ gives

$$
\Pi(m, N)-\tilde{\Pi}(m, N)=\frac{2 p+3+q^{2}-4 q}{4(4-2 q-p)}-\frac{3+2 p}{16}=\frac{3 p+4 q^{2}-10 q+4 q p+2 p^{2}}{16(4-2 q-p)} .
$$

The denominator of this expression is clearly positive and thus the sign of the difference is determined by the numerator. Denote this numerator by $d(p, q)$. 
We first show that $d(p, q)$ is negative for $m=(N+1) / 2$. Remember that, in this case, we have $p=1-2 q$ and thus

$$
d=3 p+4 q^{2}-10 q+4 q p+2 p^{2}=5-20 q+4 q^{2} .
$$

This expression is negative iff $q((N+1) / 2, N) \leq 5 / 2-\sqrt{5} \approx 0.26$, which is satisfied for all $N>5$.

Next consider any supermajority $m$ such that $(N+1) / 2<m \leq\lfloor 3 N / 4\rfloor$. For any such majority rule we have that $m \leq N-2$. Therefore, it follows that

$$
q(m, N) \geq p(m+1, N)+p(m+2, N)=p(m+1, N)\left(1+\frac{N-m-1}{m+1}\right)=p(m, N) \frac{N-m}{m} \frac{N}{m+1} .
$$

Notice that the last term in this expression is decreasing in $m$. Thus we may write $p \leq(q\lfloor 3 N / 4\rfloor\lceil 3 N / 4\rceil) /((N-\lfloor 3 N / 4\rfloor) N)$. Using Lemma 5 , it can be shown that the right-hand side of this last inequality is smaller than $(12 / 5) q \cdot{ }^{25}$

Next observe that the fact that $m$ is a supermajority rule implies that $1 / 2 \geq p(m-$ $1, N) / 2+p(m, N)+q(m, N) \geq 3 p(m, N) / 2+q(m, N)$. Combining this observation with the preceding one, we obtain $p \leq \min \{(1-2 q) / 3,12 q / 5\}$, or equivalently,

$$
p \leq\left\{\begin{array}{cl}
12 q / 5 & \text { if } q \leq 5 / 46 \\
(1-2 q) / 3 & \text { if } q>5 / 46 .
\end{array}\right.
$$

Notice that $d(12 q / 5, q)=-(14 / 5-628 q / 25) q<0$ for all $q \leq 5 / 46$ and $d((1-2 q) / 3, q)=$ $\left(11-104 q+20 q^{2}\right) / 9<0$ for all $5 / 46<q \leq 1 / 2$. Since $d(p, q) \leq d(\min \{(1-$ $2 q) / 3,12 q / 5\}, q)$, this proves the first claim.

As for the second statement, observe that a sufficient condition for the numerator of (24) to be positive is $3 p>10 q$. From Lemma 4, we have $\frac{q}{p} \leq \frac{N-m}{2 m-N}$ so that for all $m \geq \frac{13}{16} N$, the numerator of $(24)$ is positive.

${ }^{25}$ If $N=7,11,15, \ldots$, then $12 / 5-(\lfloor 3 N / 4\rfloor\lceil 3 N / 4\rceil) /((N-\lfloor 3 N / 4\rfloor) N)=(N+(N-1) / 2+8) / 10 N>0$. 


\section{References}

Aghion, P. and P. Bolton (2003). Incomplete social contracts. Journal of the European Economic Association 1(1), 38-67.

Austen-Smith, D. and J. Banks (1996). Information aggregation, rationality, and the condorcet jury theorem. American Political Science Review 90, 34-45.

Bond, P. and H. Eraslan (2007). Strategic voting over strategic proposals. University of Pennsylvania.

Buchanan, J. and G. Tullock (1962). The calculus of consent. University of Michigan Press.

Caplin, A. and B. Nalebuff (1988). On 64\%-majority rule. Econometrica 56, 787-814.

Dal Bo, E. (2006). Committees with supermajority voting yield commitment with flexibility. Journal of Public Economics 90, 573-599.

Diermeier, D. and R. Myerson (1999). Bicameralism and its consequences for the internal organization of legislatures. American Economic Review 89, 1182-1196.

Feddersen, T. and W. Pesendorfer (1996). The swing voter's curse. American Economic Review 86, 408-424.

Feddersen, T. and W. Pesendorfer (1998). Convicting the innocent: The inferiority of unanimous jury verdicts under strategic voting. American Political Science Review 92, 23-35.

Fernandez, R. and D. Rodrik (1991). Resistance to reform: Status quo bias in the presence of individual-specific uncertainty. American Economic Review 81, 114655.

Glazer, A. (1989). Politics and the choice of durability. American Economic Review 79(5), 1207-13.

Gradstein, M. (1999). Optimal taxation and fiscal constitution. Journal of Public Economics 72, 471-485.

Guttman, J. (1998). Unanimity and majority rule: the calculus of consent reconsidered. European Journal of Political Economy 14, 189-207.

Messner, M. and M. K. Polborn (2004). Voting on majority rules. Review of Economic Studies 71(1), 115-32.

Nitzan, S. and J. Paroush (1985). Collective Decision Making. Cambridge University Press.

Strulovici, B. (2007). Social choice with uncertain preferences. University of Oxford. 
Tullock, G. (1998). Reply to Guttman. European Journal of Political Economy 14, $215-218$. 